\title{
Nano-Array Integrated Structured Catalysts: A New Paradigm upon Conventional Wash-Coated Monolithic Catalysts?
}

\author{
Junfei Weng ${ }^{\dagger}$, Xingxu Lu ${ }^{\dagger}$ and Pu-Xian Gao * \\ Department of Materials Science and Engineering \& Institute of Materials Science, University of Connecticut, \\ Storrs, CT 06269-3136, USA; junfei.weng@uconn.edu (J.W.); xingxu.lu@uconn.edu (X.L.) \\ * Correspondence: puxian.gao@uconn.edu \\ † These authors contributed equally to this work.
}

Received: 14 May 2017; Accepted: 17 August 2017; Published: 28 August 2017

\begin{abstract}
The monolithic catalyst, namely the structured catalyst, is one of the important categories of catalysts used in various fields, especially in catalytic exhaust after-treatment. Despite its successful application in conventional wash-coated catalysts in both mobile and stationary catalytic converters, washcoat-based technologies are facing multi-fold challenges, including: (1) high Pt-group metals (PGM) material loading being required, driving the market prices; (2) less-than ideal distribution of washcoats in typically square-shaped channels associated with pressure drop sacrifice; and (3) far from clear correlations between macroscopic washcoat structures and their catalytic performance. To tackle these challenges, the well-defined nanostructure array (nano-array)-integrated structured catalysts which we invented and developed recently have been proven to be a promising class of cost-effective and efficient devices that may complement or substitute wash-coated catalysts. This new type of structured catalysts is composed of honeycomb-structured monoliths, whose channel surfaces are grown in situ with a nano-array forest made of traditional binary transition metal oxide support such as $\mathrm{Al}_{2} \mathrm{O}_{3}, \mathrm{CeO}_{2}, \mathrm{Co}_{3} \mathrm{O}_{4}, \mathrm{MnO}_{2}, \mathrm{TiO}_{2}$, and $\mathrm{ZnO}$, or newer support materials including perovskite-type $\mathrm{ABO}_{3}$ structures, for example $\mathrm{LaMnO}_{3}, \mathrm{LaCoO}_{3}, \mathrm{LaNiO}$, and $\mathrm{LaFeO}_{3}$. The integration strategy parts from the traditional washcoat technique. Instead, an in situ nanomaterial assembly method is utilized, such as a hydro (solva-) thermal synthesis approach, in order to create sound structure robustness, and increase ease and complex-shaped substrate adaptability. Specifically, the critical fabrication procedures for nano-array structured catalysts include deposition of seeding layer, in situ growth of nano-array, and loading of catalytic materials. The generic methodology utilization in both the magnetic stirring batch process and continuous flow reactor synthesis offers the nano-array catalysts with great potential to be scaled up readily and cost-effectively. The tunability of the structure and catalytic performance could be achieved through morphology and geometry adjustment and guest atoms and defect manipulation, as well as composite nano-array catalyst manufacture. Excellent stabilities under various conditions were also present compared to conventional wash-coated catalysts.
\end{abstract}

Keywords: nanostructures; nano-array structured catalysts; wash-coat structured catalysts; wet chemical synthesis; tunable structure and performance; stability

\section{Introduction}

Monolithic catalysts have been invented and successfully implemented in many applications, including fine chemical processing, clean energy combustion, and environmental catalysis [1]. Since the 1960s, the automotive and energy industries have witnessed the rapid development and implementation of various monolithic devices in vehicles and stationary power plants. This technology evolution process is closely associated with the increasingly stringent regulations with respect to the 
control of exhaust emissions such as carbon monoxide $(\mathrm{CO})$, hydrocarbons $\left(\mathrm{HC}_{\mathrm{s}}\right)$, nitrogen oxide $\left(\mathrm{NO}_{\mathrm{x}}\right)$, and particulate matter $(\mathrm{PM})$, therefore signaling the necessity for advanced catalysts [2,3].

Generally, the fabrication of the monolithic catalysts starts from the honeycomb substrate [4]. Cordierite, with the nominal composition of $2 \mathrm{MgO} \cdot 5 \mathrm{SiO}_{2} \cdot 2 \mathrm{Al}_{2} \mathrm{O}_{3}$, is the most common monolithic substrate material, due to its low thermal expansion coefficient, high thermal shock resistance, relatively high porosity, and good mechanical durability [5]. Owing to these merits, cordierite is able to survive various automotive and stationary exhaust conditions. Besides, cordierite monoliths exhibit low pressure drop because of the high cell density and large open frontal area, leading to excellent adaptability to large flow rate working conditions.

The relatively low surface area of the cordierite monolith makes it necessary to integrate an additional support layer, known as the washcoat, for further deposition and loading of active catalysts. However, the quality of the deposited washcoat is affected by many factors, including the particle size and properties of support materials, as well as the concentration, $\mathrm{pH}$ value and viscosity of the washcoat slurry, etc. [6]. Cracks are usually present within the wash-coated layers, which usually result from solvent evaporation during thermal processes, the difference in thermal expansion coefficients of cordierite and the washcoat, and the weak adhesion of different layers on monolith surface due to their electrostatically incompatible nature. The problem of cracks can be mitigated by adding binders and additives, but more parameters would be introduced into the manufacturing process, resulting in a much more complex system. The thickness of the wash-coated layer is a critical parameter for catalyst performance as well. A thinner washcoat provides an inadequate surface area for the deposition of a catalytically active phase, while a thicker washcoat usually renders low mass diffusion and causes a high pressure drop. Finally, the non-uniform wash-coated layer makes it difficult to determine the relationships between the catalyst structure, porosity, crystal orientation, and reaction kinetics within the gas-solid interfaces and the catalytic performance.

The extrusion of support material and the catalyst itself together into a monolithic type is an alternative to depositing catalysts on the monolithic support. Such catalysts, namely integral monolithic catalysts, could be distributed uniformly throughout the monolith. Although the total amount of available catalysts in an integral monolith could be larger than the wash-coated ones, its fabrication process could be much more specialized, with additional specific extruders required, and the necessary procedures need to be optimized to maximize the effectiveness of the catalysts [6,7].

The nanostructure array (nano-array)-integrated monolithic catalyst has been investigated by many researchers as a new class of structured catalysts or reactors to tackle and potentially address the practical catalyst issues by fully utilizing their advantageous merits [8-10]. For example, in terms of environmental remediation, the monolithic devices decorated by one-dimensional (1D) nano-array assembly have been identified to improve the spatial utilization efficiency in a limited space, which can promote the interactions between catalysts and toxic gases. On the other hand, from a manufacturing perspective, the fabrication of nano-array-integrated catalysts with fewer post-processing steps and no additional binders is a promising cost-effective route for producing advanced catalysts.

In this article, we present a comprehensive overview of the recent progress in the synthesis of various types of nano-array such as nanowires, nanotubes, and nanorods onto bare monolithic substrates, focusing on the preparation and fabrication methods, tunability of morphology and related performance, and the stability properties of various catalysts. The structural and chemical characteristics of this novel catalysts exhibit surprising advantages of excellent robustness, high material utilization efficiency, and tunable catalytic functions at low temperature.

\section{Preparation and Fabrication}

\subsection{Conventional Wash-Coating Procedure}

In order to increase the surface area for active phase deposition, and promote the interaction between gas and catalysts, it is necessary to deposit firstly a layer of oxides on the bare honeycomb 
monoliths. Compared to the pore-filling method, slurry coating is one of the most common methods for wash-coating. Nijhuis et al. [7] reviewed and described thoroughly the principles of each step during wash-coating procedures. A slurry with suspended particles is prepared, where elative large particles such as binding agents are present to provide sufficient adhesion for the coated layer. The monolith is then dipped with the excess liquid shaken out using pressurized air. The monolith is dried horizontally in static air while continuously being rotated around its axis. Finally, the monoliths are calcined in air at a high temperature. A repeated coating process could be utilized to achieve the desired loading and thickness.

In general, the quality of the wash-coated layer is influenced by various parameters as each step involves several variables to be controlled. Similar to the step of slurry preparation, the solvent amount, the binders, organic and inorganic additives, $\mathrm{pH}$, and the rheological property of the slurry could influence the wash-coating quality. Another critical procedure is the drying step. It is very easy to form a non-uniform distribution of catalysts and cause cracks among catalysts, layers, and substrates. Thus, it is essential to control appropriate temperature, temperature ramp and cooling rates, humidity, and drying time during the drying step.

Recently, the fabrication of the nano-array based structured catalysts synthesized in our group showed the promising advantages of saving time and cost and overcoming the drawbacks of the conventional wash-coating preparation mentioned above. The basic principle of hydrothermal synthesis and extended scalable fabrication with the help of magnetic stirring and continuous flow are discussed in detail below.

\subsection{Hydrothermal Synthesis}

Hydrothermal synthesis is a facile and widely used approach for the synthesis of nano-arrays in various substrates. In the past few years, we have successfully achieved the in situ growth of different types of metal oxide nano-arrays on the channel walls of commercial three-dimensional (3D) cordierite honeycomb substrates using hydrothermal synthesis $[9,11,12]$. Compared with the conventional wash-coating procedure, one-pot hydrothermal synthesis is a binder-free and environmentally benign process to grow nanostructures with adjustable geometry and orientation. The nucleation of as-synthesized nanostructure starts on the surface of the substrate, which usually ensures good adherence to the deposition substrates [11].

Figure 1 illustrates the fabrication process for various nano-arrays on 3D cordierite and stainless steel honeycomb substrates [9]. The substrate was $~ 1$ inch in diameter and 0.5 inches in channel length. The as-prepared monolithic nano-array catalyst demonstrated the feasibility of direct integration of nano-arrays onto the 3D substrate by solution chemistry on the laboratory scale. The photographic and microscopic characterization of four types of nano-array-based monolithic catalysts, including $\mathrm{ZnO}$ nanowire arrays, $\mathrm{TiO}_{2}$ nanorod arrays, $\mathrm{CeO}_{2}$ nanotube arrays, and $\mathrm{Co}_{3} \mathrm{O}_{4}$ nanowire arrays are shown in Figure 1 as well.

The procedures for the synthesis or integration of metal oxide nano-arrays based catalysts onto 3D monolithic substrates include (1) deposition of seeding layer; (2) in situ nucleation and growth of nano-arrays; and (3) loading of catalytic materials. The seeding and nucleation process is crucial for the synthesis of nano-arrays. The growth of metal oxide nano-arrays is usually a competition process between homogeneous nucleation and heterogeneous nucleation in solution, and heterogeneous nucleation generally has a lower activation energy barrier than homogeneous nucleation. Therefore, growth on existing seeds is more favorable than nucleation in homogeneous bulk solution for the reason that the existing seeds help bypass or lower the thermodynamic energy barrier of the nucleation step [13]. A simple dip-coating method was employed to create a seeding layer onto the channel walls of 3D monolithic substrates, and the ultrasonic vibration technique could help to improve the uniformity of the seeding layers across the channels $[12,14,15]$. For example, Figure 2 displays the schematic of the growth of $\mathrm{ZnO}$ nano-arrays on monolithic cordierite substrate from the seeding step and the uniformity comparison between the $\mathrm{ZnO}$ nano-arrays with and without ultrasonic 
vibration during the seeding procedure [14]. The enhanced uniformity of $\mathrm{ZnO}$ nanorod arrays across the channels indicated that ultrasonic vibration during the dip-coating procedure was an effective means for improving the uniformity of the seed layers, hence promoting the uniform growth of nanorods (NR).

a

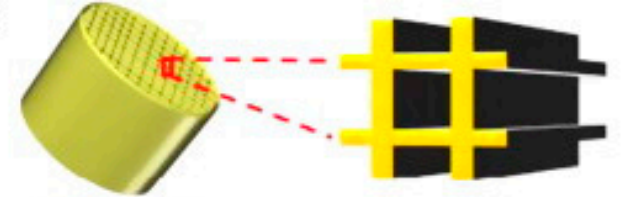

Cordierite

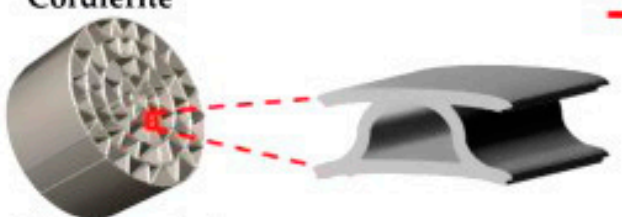

Stainless steel
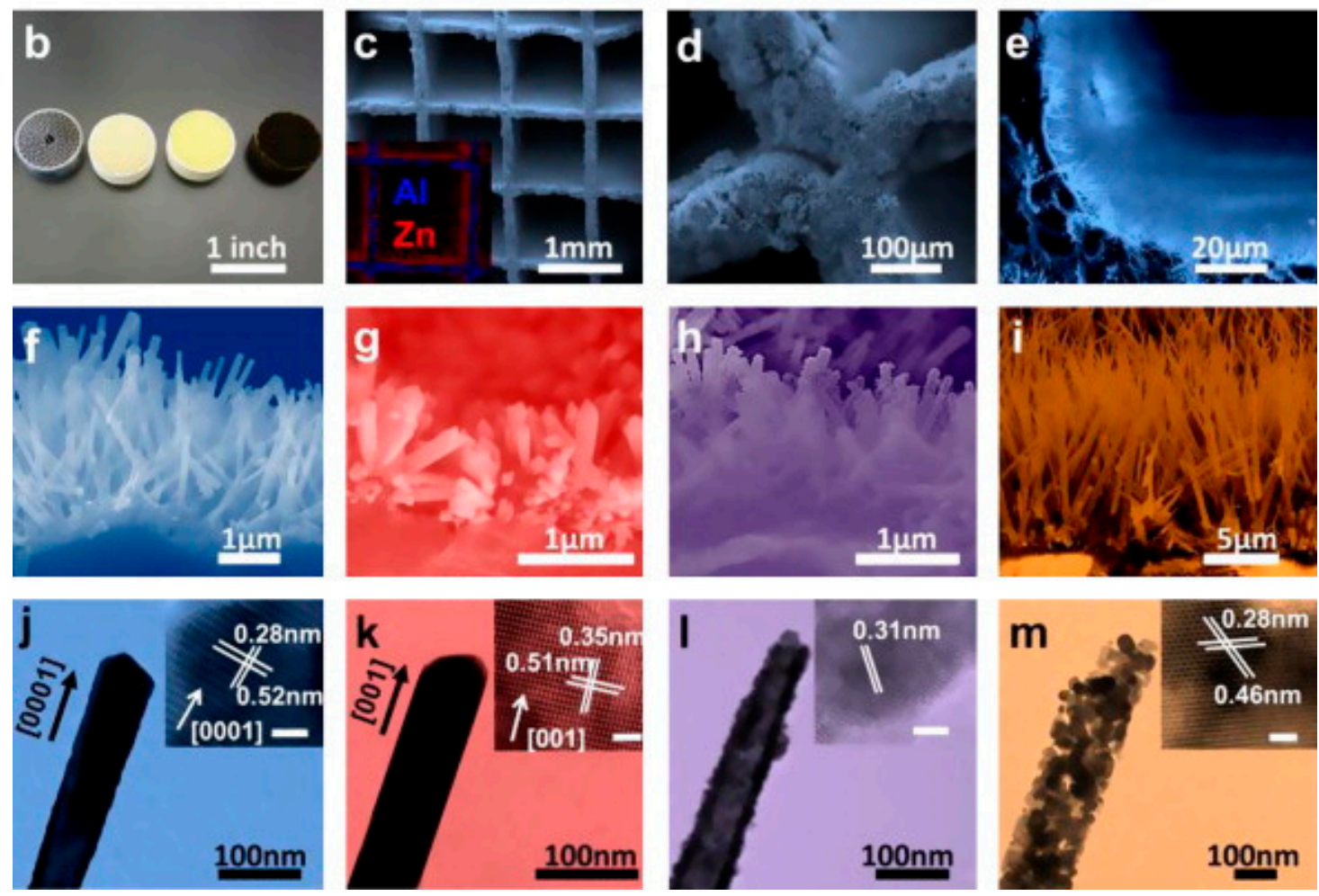

Figure 1. (a) Schematic illustration for the nano-array growth onto the three-dimensional (3D) monolithic cordierite and stainless steel substrate by hydrothermal synthesis; (b) Photographs of monolithic nano-array catalysts with various nano-arrays deposited; (c) low-magnification SEM image of a $\mathrm{ZnO}$ nano-array catalyst with inset showing the element distribution; (d,e) low magnification top and cross-sectional SEM images of nano-arrays; (f-i) cross-sectional SEM images of (f) ZnO nanowire arrays; (g) $\mathrm{TiO}_{2}$ nanorod arrays; (h) $\mathrm{CeO}_{2}$ nanotube arrays; (i) $\mathrm{Co}_{3} \mathrm{O}_{4}$ nanowire arrays; $(\mathbf{j}-\mathbf{m})$ corresponding TEM characterization of nano-arrays shown in $(\mathbf{f}-\mathbf{i})$. Reprinted with permission from [36], Copyright Elsevier, 2013. 

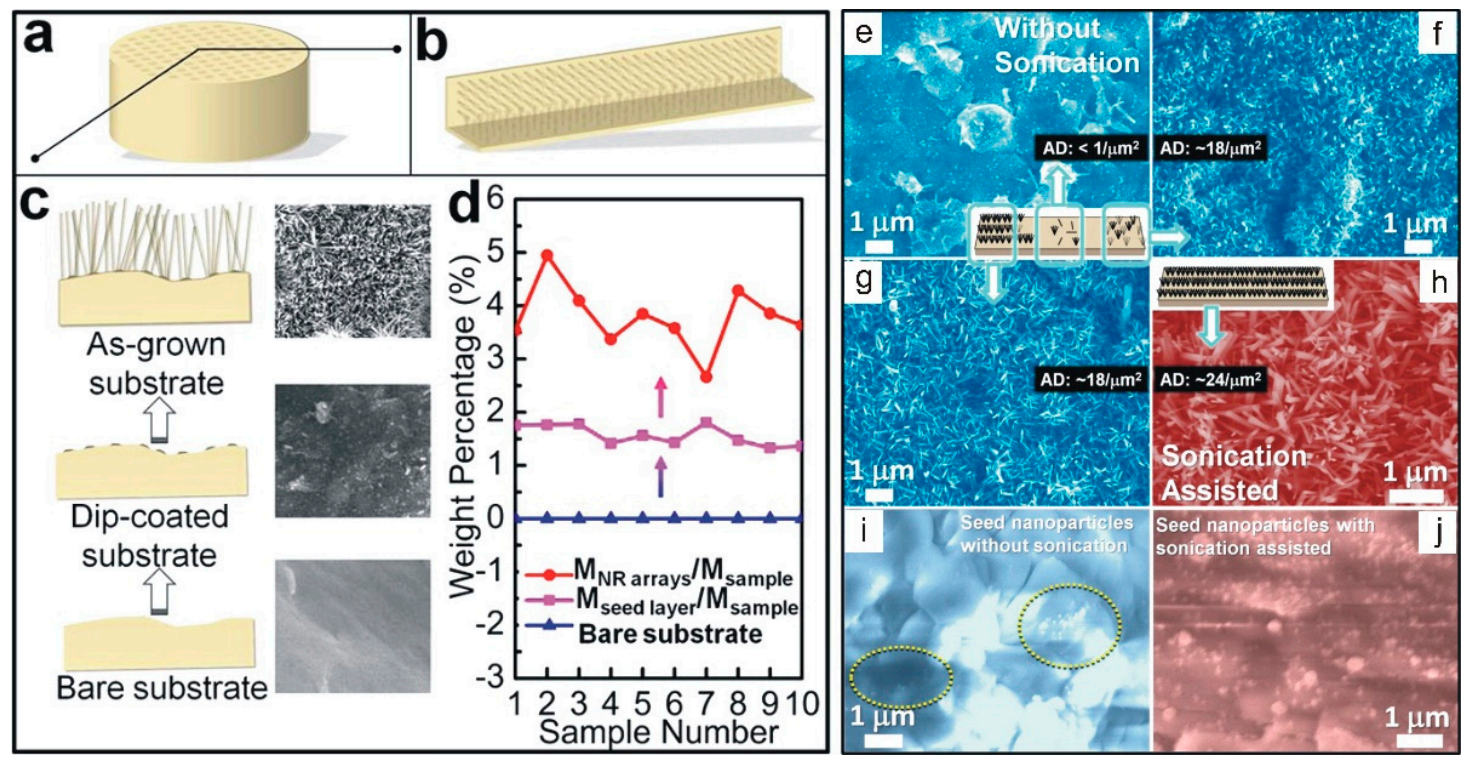

Figure 2. (a,b) Schematic illustrations of (a) monolithic cordierite substrate and (b) as-grown nanorod (NR) arrays inside each channel; (c) Cross-sectional diagram of the ZnO NR array growth process; (d) Weight percentages of seed layer and as-grown ZnO NR arrays with respect to the total masses of dip-coated and as-grown substrates, respectively. SEM images of synthesized ZnO NR arrays: (e-g) different channel regions of an as-grown sample whose seed nanoparticles were dip-coated without ultrasonic vibration; (h) ZnO NR arrays grown in the center channel surface region of an as-grown sample whose seed nanoparticles were dip-coated with ultrasonic vibration; and (f-i) seed nanoparticles dip-coated with and without ultrasonic vibration assistance. Reprinted with permission from [14], Copyright ACS, 2013.

For the procedure of nano-array growth, Teflon-lined stainless steel autoclaves and ordinary beakers are commonly used reactors for hydrothermal synthesis at elevated and low temperatures, respectively. In typical synthesis, seeded 3D monolithic substrates were immersed in prepared solutions in the reactors, and then the autoclaves or beakers were sealed and heated at the desired temperature for designed time. After the reactors cooled to room temperature, the obtained products were rinsed with distilled water or ethanol, and then dried or annealed at desired temperature. In our previous research, $\mathrm{Co}_{3} \mathrm{O}_{4}$ nanowire array-based catalysts were fabricated onto large commercial cordierite monolithic substrates following a low-temperature hydrothermal process [16-18]. As shown in Figure 3, a commercial honeycomb support of $392.5 \mathrm{~mL}$ was used for growing $\mathrm{Co}_{3} \mathrm{O}_{4}$-based nanowire arrays. Uniform coverage of nanostructures of $\sim 10 \mu \mathrm{m}$ in length was achieved throughout the 5-cm-long honeycomb channels [17]. The successful fabrication of industrially-relevant sized $\mathrm{Co}_{3} \mathrm{O}_{4}$ nano-array monolithic catalysts using hydrothermal synthesis represented an important step towards the large scale production and potential application of nano-array based monolithic catalysts.

Recently, our group have extended the in situ growth of nano-array structures on the 3D honeycomb cordierite monolithic substrates to the application of $\mathrm{TiO}_{2}$ nanorods [9]. As shown in Figure 3, the as-synthesized single crystalline $\mathrm{TiO}_{2}$ nanorod arrays were vertically and uniformly aligned on each channel wall. As reaction time proceeded, it was found that the brookite nanostructure morphology was evolved from dispersive dandelion $\mathrm{TiO}_{2}$ nanorod clusters to vertically aligned nanorod arrays, and then fully grown on the channel walls. Moreover, the $\mathrm{TiO}_{2}$ nanorod arrays, with a high surface area of up to $\sim 250 \mathrm{~m}^{2} \cdot \mathrm{g}^{-1}$, exhibit the potential of being a good platform for coating other active components. Besides nanorod and nanowire arrays, we also developed a templated method to prepare nanotube arrays. For example, due to the difficulty of direct hydrothermal synthesis of $\mathrm{CeO}_{2}$ nano-arrays, $\mathrm{ZnO}$ nanowires were grown firstly by hydrothermal method as the template, and $\mathrm{CeO}_{2}$ layers were subsequently deposited to the $\mathrm{ZnO}$ surface to form core-shell nanostructures. The followed 
hydrogen treatment could remove the $\mathrm{ZnO}$ core easily to form the $\mathrm{CeO}_{2}$ nanotubes, as $\mathrm{ZnO}$ could easily react with hydrogen at a high temperature with concurrent sublimation of metal $\mathrm{Zn}$ with a relatively low sublimation point, as shown in Figure 3iv [19].

(i)
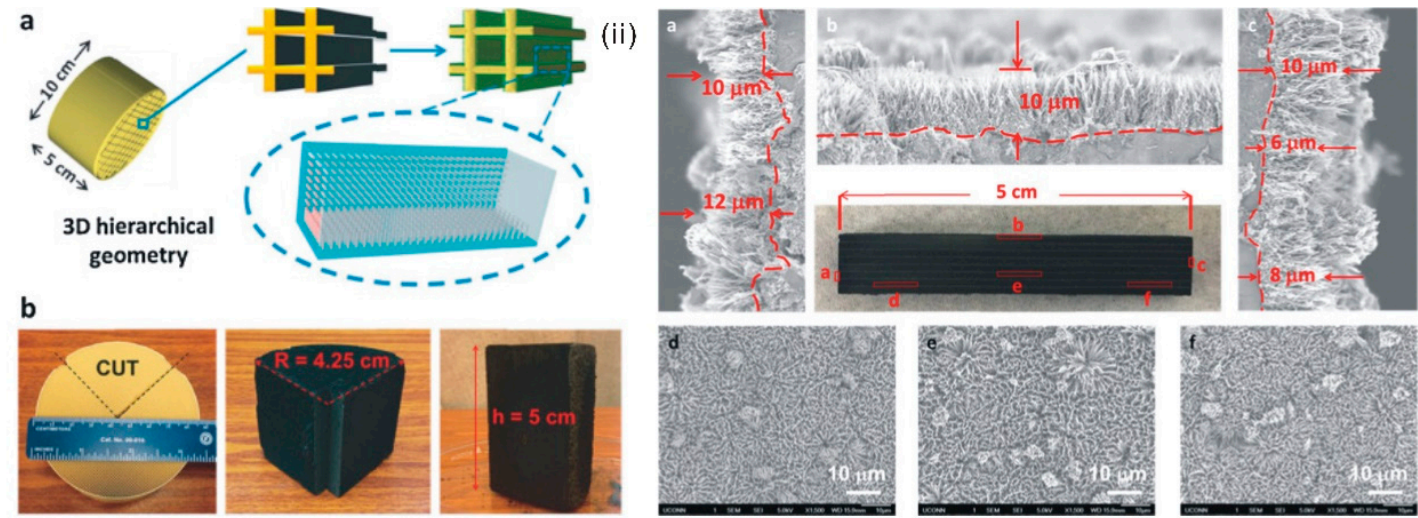

(iii) (a)
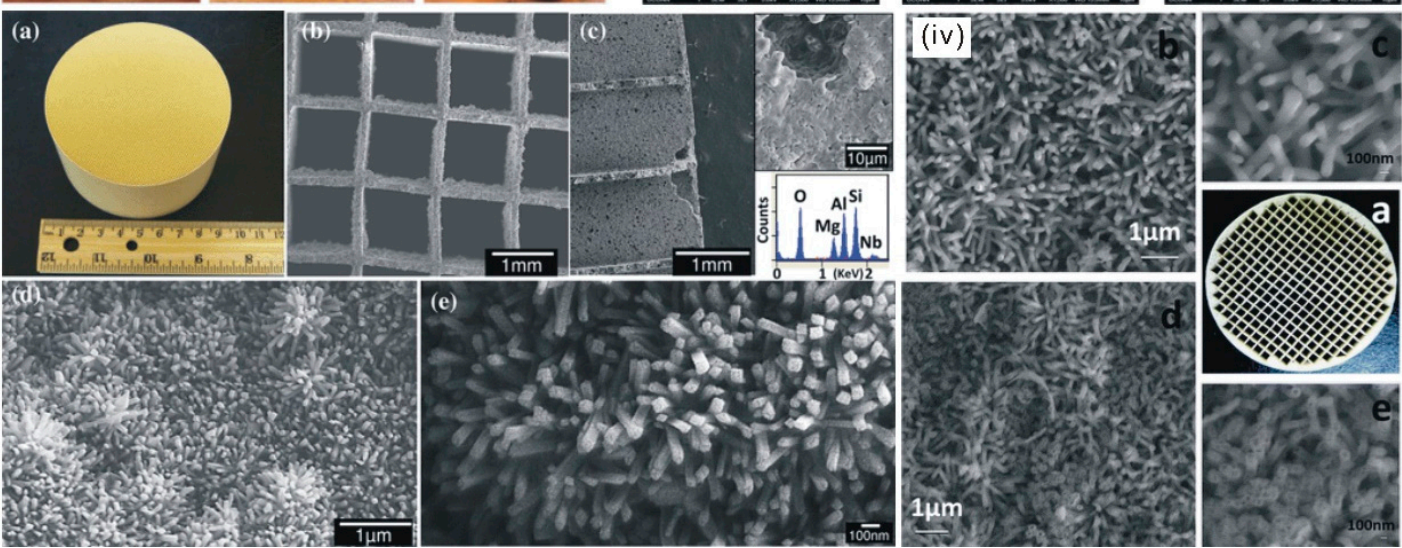

Figure 3. (i) (a) Monolithic integration of nano-arrays on commercialized honeycomb supports. (b) Dimensions of a typical piece of a monolithic nano-array catalyst; (ii) SEM images of the nano-arrays grown inside the monolithic honeycomb channels. (a-c) Cross-sectional views and $(\mathrm{d}-\mathrm{f})$ top views of $\mathrm{Co}_{3} \mathrm{O}_{4}$ nano-arrays; (iii) (a) Top-view optical and (b) SEM images of the honeycomb monolith. (c) Side-view SEM images of the honeycomb monolith and the corresponding Energy-dispersive X-ray (EDX) spectrum. (d,e) SEM images of $\mathrm{TiO}_{2}$ nanorod arrays grown on the 3D honeycomb monolith; (iv) (a) Photograph of a 3D cordierite honeycomb substrate. SEM images of ( $\mathbf{b}, \mathbf{c}) \mathrm{CeO}_{2}-\mathrm{ZnO}$ composite nano-arrays before removing the $\mathrm{ZnO}$ template and $(\mathbf{d}, \mathbf{e}) \mathrm{CeO}_{2}$ nanotube arrays after removing the $\mathrm{ZnO}$ template. Reprinted with permission from [9], Copyright RSC, 2013. Reprinted with permission from [17], Copyright Wiley, 2014. Reprinted with permission from [19], Copyright RSC, 2012.

The in situ synthesized metal-oxide nano-arrays can either exhibit catalytic function alone or, more commonly, be used as supports for loading catalytic active materials or synthesized with other active materials to make composite catalysts. For example, $\mathrm{Co}_{3} \mathrm{O}_{4}$ nano-arrays were reported to show catalytic activities for $\mathrm{NO}$ oxidation [16]. Besides, in our previous study, the Pt nanoparticles were loaded onto nano-array rooted monoliths by dip deposition of colloid Pt nanoparticles made from thermal decomposition of Pt-containing hydrochloride salts [9]. TEM characterizations of $\mathrm{Pt} / \mathrm{ZnO}$ nanorod, $\mathrm{Pt} / \mathrm{TiO}_{2}$ nanorod, $\mathrm{Pt} / \mathrm{CeO}_{2}$ nanotube, and $\mathrm{Pt} / \mathrm{Co}_{3} \mathrm{O}_{4}$ nanowires are shown in Figure 4 . The $\mathrm{Pt}$ nanoparticles of $\sim 2 \mathrm{~nm}$ were evenly dispersed on the nanowires with the $\mathrm{Pt}\{111\}$ atomic planes being exposed, as identified by the inset High-resolution transmission electron microscopy (HRTEM) images. 

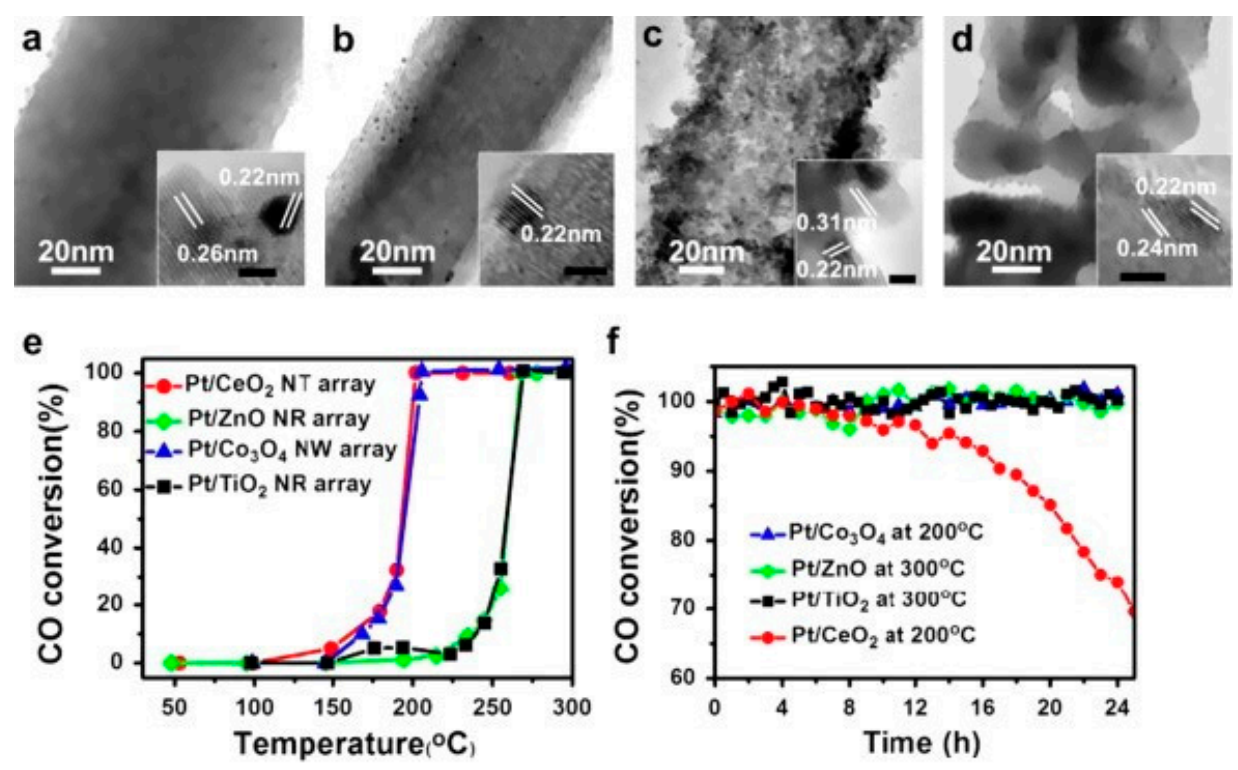

Figure 4. $\mathrm{CO}$ oxidation over Pt-loaded metal oxide nano-array-based monolithic catalysts. Low-magnification TEM images of individual Pt nanoparticle-loaded metal oxide nanostructure and its high magnification HRTEM lattice image (inset): (a) $\mathrm{ZnO}$ nanorod; (b) $\mathrm{TiO}_{2}$ nanorod; (c) $\mathrm{CeO}_{2}$ nanotube; and (d) $\mathrm{Co}_{3} \mathrm{O}_{4}$ nanowire. Scale bars in all the insets are $2 \mathrm{~nm}$; (e) CO oxidation conversion as a function of temperature over Pt-loaded $\left(\mathrm{CeO}_{2}, \mathrm{ZnO}, \mathrm{Co}_{3} \mathrm{O}_{4}\right.$, and $\left.\mathrm{TiO}_{2}\right)$ nano-array monolithic catalysts; (f) Catalytic stability of Pt-loaded $\left(\mathrm{CeO}_{2}, \mathrm{ZnO}, \mathrm{Co}_{3} \mathrm{O}_{4}\right.$, and $\left.\mathrm{TiO}_{2}\right)$ nano-array monolithic catalysts. Reprinted with permission from [36], Copyright Elsevier, 2013.

\subsection{Scalable Fabrication}

So far, we have proven the feasibility of hydrothermal synthesis for 3D integration of nano-arrays into the monolithic substrates by demonstrating that the small-sized monolithic nano-array catalyst could be synthesized successfully. However, the industrially relevant manufacturing of nano-array-based monolithic catalysts was an indispensable step toward practical application in catalyst industry. One of the main challenges for the larger-scale fabrication lies on the difficulty of the mass transfer of solution precursor and how to enable the uniform access of solution precursor throughout the long channels. The lower temperature would make the mass transfer of precursors and uniform growth of the nano-array structure more challenging [10,11].

Introducing the mechanical agitation through magnetic stirring proved a successful example for facilitating mass transport and achieving uniform coverage of the grown nano-array structures throughout the monolithic channels. Figure $5 \mathrm{a}-\mathrm{d}$ displays the $\mathrm{ZnO}$ nano-array growth with and without magnetic stirring. It was clearly observed that the nano-array coverage in the center region of cordierite channels was greatly improved when the magnetic stirring was utilized during the synthesis, as compared to the sparsely distributed $\mathrm{ZnO}$ nano-arrays without stirring [14].

On the other hand, the production efficiency of conventional batch process was usually not as prominent as the continuous flow reaction process for scaled-up industrial processing. A hydrothermal-based continuous flow synthesis (CFS) has been developed in our group to enable stable and consecutive mass transport throughout the entire channels, as shown in Figure 5e. The solution precursor was circulated within the flow reactor tubing system, being pre-heated before entering the reactor and then cooled down after coming out. Such a close-loop continuous circulation system effectively suppressed the growth of homogeneously formed nuclei by confining the crystal growth within the honeycomb substrate, giving rise to an enhanced average growth rate and improved precursor utilization efficiency. The pumping flow rate was found to be a crucial parameter that not only determined the nano-array's distribution uniformity but also sustained a stable and fast growth rate. Figure $5 \mathrm{f}$ shows the uniformly 
distributed $\mathrm{ZnO}$ nanorod array on a monolithic substrate fabricated by continuous flow synthesis [12]. The innovative CFS process could be applied for synthesis of various other 3D nano-array-integrated monolithic devices.
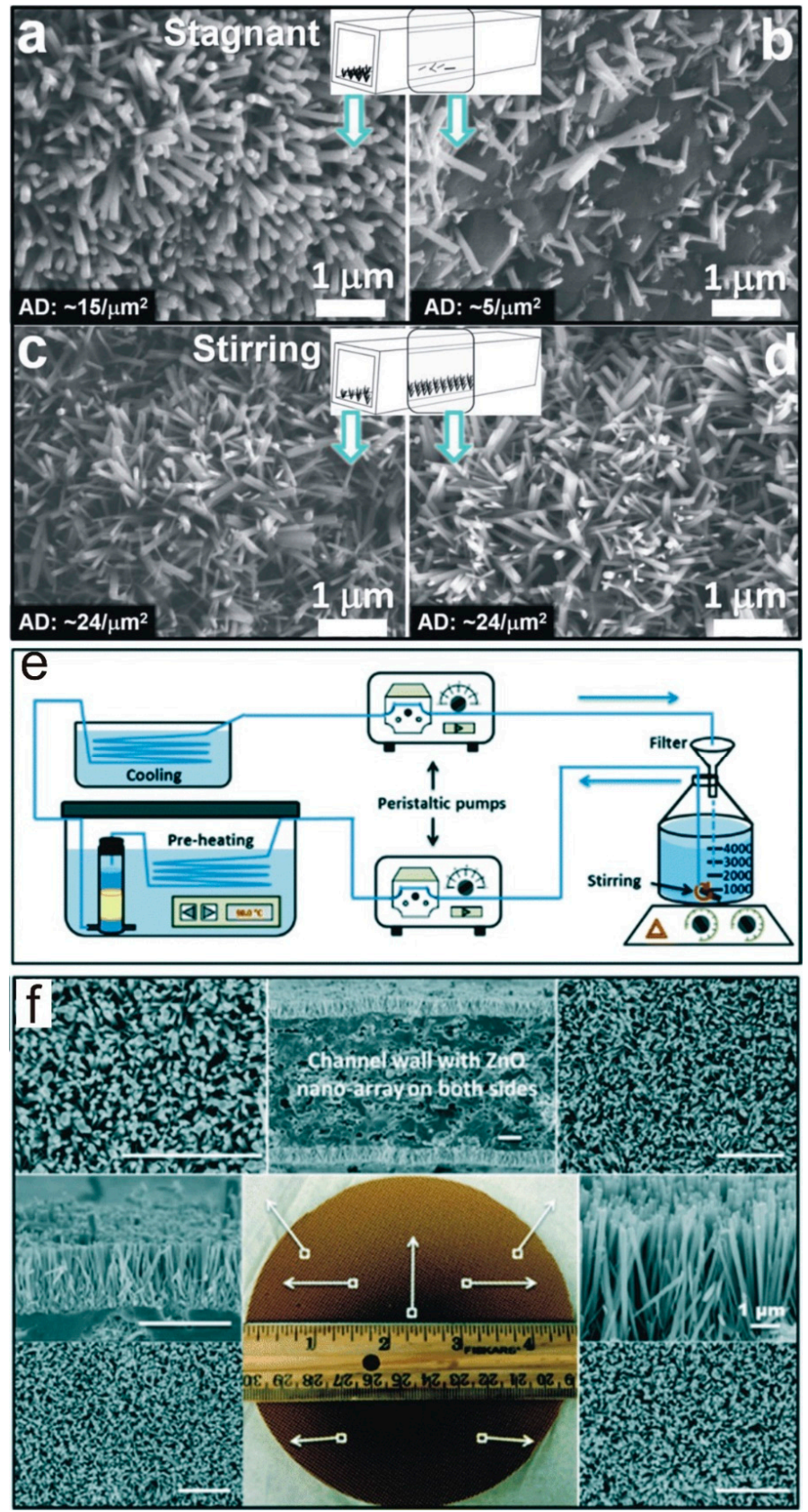

Figure 5. Mechanical agitation-assisted hydrothermal growth of ZnO nano-arrays. (a-d) SEM images of the as-grown nano-arrays at the end and center regions of cordierite channels after synthesis with and without mechanical agitation; (e) Experimental setup of the continuous flow synthesis for scalable integration of $\mathrm{ZnO}$ nanorod array on 3D honeycomb cordierite; (f) Uniformly distributed $\mathrm{ZnO}$ nanorod array on a monolithic substrate fabricated by continuous flow synthesis. Reprinted with permission from [12], Copyright RSC, 2016. Reprinted with permission from [14], Copyright ACS, 2013.

Based on the comprehensive knowledge of synthesizing the in situ grown nano-array structured catalysts with the control flexibility in our groups, a series of properties were characterized as well to demonstrate the desired performance and design tunability, which are illustrated in the following section. 


\section{Tunable Structural and Catalytic Performance}

One of the most important advantages of nano-array-based monolithic catalysts is the well-defined geometry distinct from the usually randomly distributed microstructure in the wash-coated particulates. This provides a new pathway to precisely build and optimize the structure-property correlations for catalysts in the perspective of materials science. In this section, we will first introduce the general catalytic performance of nano-array-based monolithic catalysts, and then employ several examples to demonstrate the controllability of the tunable catalyst performance by manipulating nano-array geometry and morphology, crystal structure and chemical composition. We intend to elucidate the structure-property relationship in nano-array catalysis and provide some insightful guidance of rational design of nano-array based catalysts for auto exhaust oxidations

\subsection{General Catalytic Performance of Nano-Array Based Monolithic Catalysts}

Figure $4 \mathrm{e}, \mathrm{f}$ shows the catalytic performance for $\mathrm{CO}$ oxidation by the hydrothermally integrated monolithic nano-arrays loaded with Pt nanoparticles (1 wt. \% on nano-arrays) [9]. The average size of the loaded $\mathrm{Pt}$ nanoparticles was $\sim 2 \mathrm{~nm}$ and they were well-dispersed on nanowires with the exposed crystal planes of $\mathrm{Pt}\{111\}$ as identified by the HRTEM analysis. The catalytic activity for CO oxidation was tested in the reaction feed of $1 \% \mathrm{CO}, 10 \% \mathrm{O}_{2}$ balanced by argon with a space velocity of $45,000 \mathrm{~h}^{-1}$. The light-off temperatures $\mathrm{T}_{50}$ (temperature for $50 \%$ conversion of total $\mathrm{CO}$ ) for $\mathrm{CO}$ oxidation of Pt-loaded $\mathrm{CeO}_{2}, \mathrm{ZnO}, \mathrm{Co}_{3} \mathrm{O}_{4}$, and $\mathrm{TiO}_{2}$ nano-array-based monolithic catalysts were $193^{\circ} \mathrm{C}, 260^{\circ} \mathrm{C}, 195^{\circ} \mathrm{C}$ and $258^{\circ} \mathrm{C}$, respectively. All of these monolithic catalysts were able to achieve complete $\mathrm{CO}$ conversion below $300{ }^{\circ} \mathrm{C}$. The better $\mathrm{CO}$ oxidation performance from the $\mathrm{Pt} / \mathrm{CeO}_{2}$ and $\mathrm{Pt} / \mathrm{Co}_{3} \mathrm{O}_{4}$ nano-array-based catalysts was due to the catalytic promotion effect of $\mathrm{Co}_{3} \mathrm{O}_{4}$ and $\mathrm{CeO}_{2}$ nanostructures with catalytic activity themselves.

\subsection{Morphology and Geometry Adjustment}

The nano-array configuration with well-defined geometry and orientation provides a new pathway to study the structure-property relationship of catalysts on a nano scale. Due to the rapid development of solution chemistry, it has become a relatively mature process to produce nanomaterials with controlled shape, morphology and orientations. It has been widely reported the nanocrystals with different controlled geometries were able to exhibit different catalytic activities [20]. In this section, we will present two examples of $\mathrm{Co}_{3} \mathrm{O}_{4}$ and $\mathrm{ZnO}$ nano-arrays to demonstrate the controllability of the catalytic activity of the metal oxide nano-array based monolithic catalysts.

Cobalt-based oxides have been studied as more cost-effective materials compared to noble metal based catalysts for different oxidation applications [21-24]. So far, we have prepared the $\mathrm{Co}_{3} \mathrm{O}_{4}$ nano-array-based monolithic catalysts using different cobalt precursors, such as cobalt acetate tetrahydrate $\left(\mathrm{Co}\left(\mathrm{C}_{2} \mathrm{H}_{3} \mathrm{O}_{2}\right)_{2} \cdot 4 \mathrm{H}_{2} \mathrm{O}\right.$, noted as $\left.\mathrm{CA}\right)$, cobalt nitrate hexahydrate $\left(\mathrm{Co}\left(\mathrm{NO}_{3}\right)_{2} \cdot 6 \mathrm{H}_{2} \mathrm{O}\right.$, noted as $\mathrm{CN})$ and cobalt chloride hexahydrate $\left(\mathrm{CoCl}_{2} \cdot 6 \mathrm{H}_{2} \mathrm{O}\right.$, noted as $\left.\mathrm{CC}\right)$, for low temperature oxidation of NO [16]. Figure 6a-c shows the SEM images of prepared $\mathrm{Co}_{3} \mathrm{O}_{4}$ nano-arrays. The X-ray diffraction patterns in Figure 6d confirmed that all three types of nanowire arrays prepared from different cobalt precursors corresponded to $\mathrm{Co}_{3} \mathrm{O}_{4}$. As shown in Figure $6 \mathrm{e}, \mathrm{f}$, the $\mathrm{Co}_{3} \mathrm{O}_{4}$ nano-arrays prepared from $\mathrm{Co}\left(\mathrm{NO}_{3}\right)_{2} \cdot 6 \mathrm{H}_{2} \mathrm{O}$ and $\mathrm{Co}\left(\mathrm{C}_{2} \mathrm{H}_{3} \mathrm{O}_{2}\right)_{2} \cdot 4 \mathrm{H}_{2} \mathrm{O}$ showed good $\mathrm{NO}$ oxidation performance, with $80 \%$ conversion of $\mathrm{NO}$ to $\mathrm{NO}_{2}$ at the temperature below $300{ }^{\circ} \mathrm{C}$, which were comparable to the platinum-group metal (PGM) catalysts. Moreover, the NO oxidation activity of nano-arrays was found to be better than that of the powder-form catalysts prepared from the same hydrothermal process. $\mathrm{Co}_{3} \mathrm{O}_{4}$ nano-arrays synthesized from $\mathrm{Co}\left(\mathrm{C}_{2} \mathrm{H}_{3} \mathrm{O}_{2}\right)_{2} \cdot 4 \mathrm{H}_{2} \mathrm{O}$ and $\mathrm{Co}\left(\mathrm{NO}_{3}\right)_{2} \cdot 6 \mathrm{H}_{2} \mathrm{O}$ exhibited the highest $\mathrm{NO}$ oxidation conversion at relatively low temperatures. Overall, the prepared $\mathrm{Co}_{3} \mathrm{O}_{4}$ nano-array-based catalysts demonstrated excellent low temperature activity for NO oxidation (and $\mathrm{NO}_{2}$ formation), which could greatly benefit the selective catalytic reduction (SCR) and $\mathrm{NO}_{\mathrm{x}}$ storage and reduction (NSR) processes for $\mathrm{NO}_{\mathrm{x}}$ treatments. 

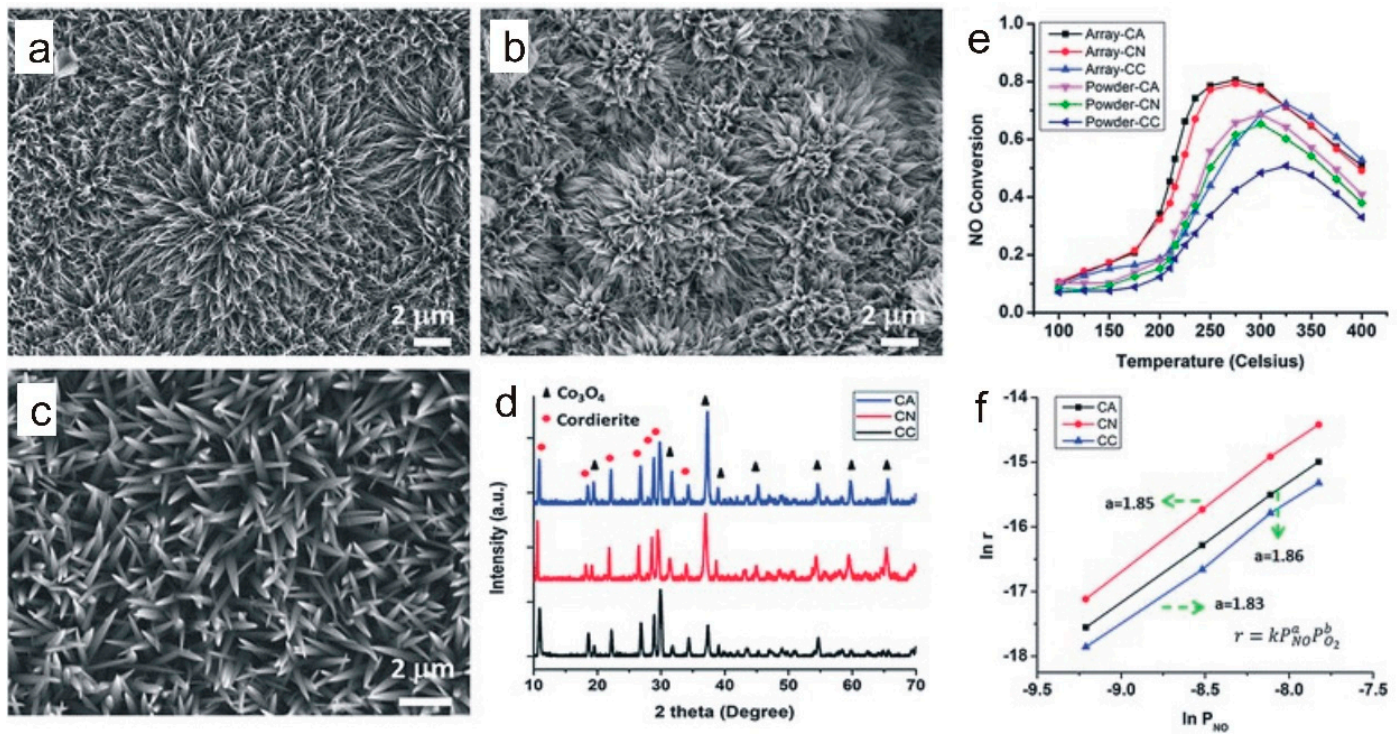

Figure 6. SEM images of $\mathrm{Co}_{3} \mathrm{O}_{4}$ nano-arrays made from (a) cobalt nitrate; (b) cobalt acetate and (c) cobalt chloride; (d) X-ray diffraction patterns of $\mathrm{Co}_{3} \mathrm{O}_{4}$ nano-arrays on cordierite honeycomb substrates; (e) $\mathrm{NO}$ oxidation performance of catalytic $\mathrm{Co}_{3} \mathrm{O}_{4}$ nano-arrays and powders prepared from different precursors; (f) Reaction rates $\mathrm{r}_{\mathrm{NO}}$ measured at $100{ }^{\circ} \mathrm{C}$ as a function of $\mathrm{CO}$ or $\mathrm{O}_{2}$ concentration over different $\mathrm{Co}_{3} \mathrm{O}_{4}$ nanowire arrays. Reprinted with permission from [16], Copyright RSC, 2013. CA: cobalt acetate tetrahydrate; $\mathrm{CN}$ : cobalt nitrate hexahydrate; $\mathrm{CC}$ : cobalt chloride hexahydrate.

Table 1 summarizes the results of X-ray photoelectron spectroscopy (XPS) spectra and relative intensity of primary crystal planes of $\mathrm{Co}_{3} \mathrm{O}_{4}$ in X-Ray Diffraction (XRD) patterns [16]. Samples synthesized by $\mathrm{Co}\left(\mathrm{NO}_{3}\right)_{2} \cdot 6 \mathrm{H}_{2} \mathrm{O}$ and $\mathrm{Co}\left(\mathrm{C}_{2} \mathrm{H}_{3} \mathrm{O}_{2}\right)_{2} \cdot 4 \mathrm{H}_{2} \mathrm{O}(\mathrm{CA}$ and $\mathrm{CN})$ showed similar performance for $\mathrm{NO}$ conversion, which were better than the catalytic performance of the samples from $\mathrm{CoCl}_{2} \cdot 6 \mathrm{H}_{2} \mathrm{O}$ (CC). The enhanced catalytic activity of $\mathrm{CA}$ and $\mathrm{CN}$ was attributed to the more exposure of $\{220\}$ planes, which was demonstrated by the relatively higher intensity of $\{220\}$ in XRD patterns. $\mathrm{Co}^{3+}$ ions on the $\{220\}$ crystal planes are the active sites for catalytic NO oxidation, and the higher quantity of $\mathrm{Co}^{3+}$ ions in CA and $\mathrm{CN}$ was also verified by the ratio of $\mathrm{Co}^{3+} / \mathrm{Co}^{2+}$ on the catalyst surface examined by XPS.

Table 1. Reaction kinetics parameters, $\mathrm{Co}^{3+} / \mathrm{Co}^{2+}$ ratio calculated from deconvoluted X-ray photoelectron spectroscopy (XPS) spectra and XRD relative intensity of primary crystal planes of $\mathrm{Co}_{3} \mathrm{O}_{4}[16]^{\mathrm{a}}$.

\begin{tabular}{|c|c|c|c|c|c|c|c|c|}
\hline \multirow[t]{2}{*}{ Catalyst } & \multicolumn{2}{|c|}{ Kinetics Parameters } & \multirow{2}{*}{$\frac{\mathrm{XPS}}{\mathrm{Co}^{3+} / \mathrm{Co}^{2+}}$} & \multicolumn{5}{|c|}{ Diffraction Planes } \\
\hline & $\underset{\left(\mathrm{mol} \cdot \mathrm{g}^{-1} \cdot \mathrm{s}^{-1}\right)}{\mathrm{k}_{0}}$ & $\begin{array}{c}\mathrm{Ea} \\
\left(\mathrm{kJ} \cdot \mathrm{mol}^{-1}\right)\end{array}$ & & $\{111\}$ & $\{220\}$ & $\{311\}$ & $\{222\}$ & $\{400\}$ \\
\hline CA & 5433 & 20.6 & 1.85 & 1.01 & 0.73 & 0.50 & 0.83 & 0.72 \\
\hline $\mathrm{CN}$ & 5102 & 20.1 & 1.85 & 0.98 & 0.71 & 0.65 & 1.50 & 0.73 \\
\hline $\mathrm{CC}$ & 3840 & 20.1 & 1.40 & 1.10 & 0.57 & 0.32 & 1.67 & 0.40 \\
\hline
\end{tabular}

${ }^{a}$ Reaction kinetics parameters were obtained from Arrhenius plots in the temperature range of $110-150{ }^{\circ} \mathrm{C}$ with the feed gas stream of $500 \mathrm{ppm} \mathrm{NO}$ and $10 \% \mathrm{O}_{2}$ under the space velocity of $\sim 50,000 \mathrm{~h}^{-1}$.

In addition to the enclosed crystal planes, the geometric size of the nanostructures of the nano-array integrated monolithic catalysts can also be tuned. In this part, $\mathrm{Pt}$ loaded $\mathrm{ZnO}$ nano-arrays were employed as an example to demonstrate how the catalytic performance can be effected by the size and the crystal planes of the nano-arrays [9]. The catalytically inert $\mathrm{ZnO}$ nano-array supports provided a good platform to exemplify the roles of the enclosed crystal surfaces and interfaces with catalytically active $\mathrm{Pt}$ nanoparticles. By tuning the aspect ratio of $\mathrm{ZnO}$ nano-arrays, we successfully 
obtained nanowires and nanoplates with the dominant plane of $\{10 \overline{1} 0\}$ and $\{0001\}$, respectively. Furthermore, different lengths of $\mathrm{ZnO}$ nanowires from $1 \mu \mathrm{m}$ to $5 \mu \mathrm{m}$ were synthesized by controlling the reaction time. Figure 7 displays the SEM and TEM images of $\mathrm{ZnO}$ nano-arrays and nanoplates with different morphologies and lengths in comparison with the wash-coated $\mathrm{ZnO}$ powder-based monolith. With equivalent amount of $\mathrm{Pt}$ catalysts loaded onto these $\mathrm{ZnO}$ nano-arrays, the structural effects of the $\mathrm{ZnO}$ supports on the catalytic performance were investigated through $\mathrm{CO}$ oxidation reactions. Figure 8 present the light-off curves of different nano-array supported Pt nanoparticles, from which we can see that the Pt nanoparticles anchored on longer $\mathrm{ZnO}$ nanowires exhibit better activity and achieve complete $\mathrm{CO}$ conversion at lower temperature. On the other hand, the catalytic performance of $\mathrm{Pt} / \mathrm{ZnO}$ nanowires was not as good as that of $\mathrm{Pt}$ supported on $\mathrm{ZnO}$ nanoplates, and that can be attributed to the interfacial promotion effects by the synergy between Pt nanoparticles and the polar $\mathrm{ZnO}\{0001\}$ surfaces which were dominant in $\mathrm{ZnO}$ nanoplates. Another interesting result is that the catalytic activity of $\mathrm{Pt}$ supported on all $\mathrm{ZnO}$ nano-arrays was better than that of $\mathrm{Pt} / \mathrm{ZnO}$ wash-coated powders. Therefore, by controlling the length of nano-arrays and support structures, the catalytic activity of nano-array-based catalysts could be rationally adjusted, which opened up new pathways to tune the collective catalyst performance. The above mentioned investigations could serve as a perfect example of rational design of nanomaterials and adjusting catalyst activity based on structure-property correlations.

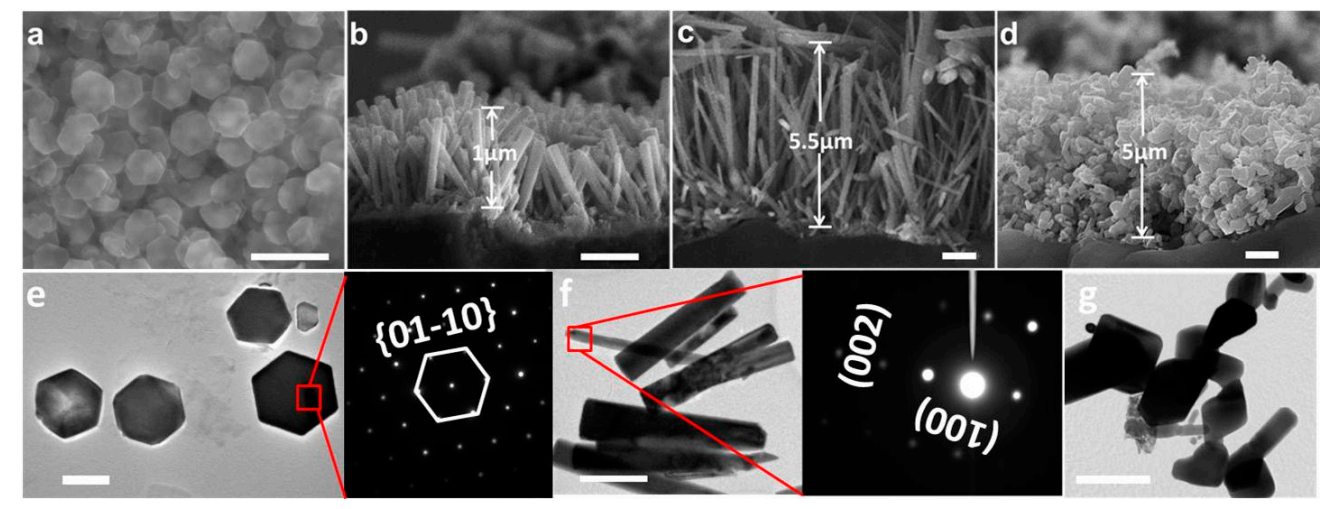

Figure 7. Electron microscopy characterization of $\mathrm{ZnO}$ nano-arrays with different shapes and sizes. SEM images of (a) nanoplates; (b) 1- $\mu \mathrm{m}$ nanowire; (c) 5- $\mu \mathrm{m}$ nanowire; (d) wash-coated $\mathrm{ZnO}$ powders. TEM images of (e) ZnO nanoplate with inset electron diffraction pattern; (f) ZnO nanowires with inset electron diffraction pattern; (g) ZnO powders. Reprinted with permission from [36], Copyright Elsevier, 2013.
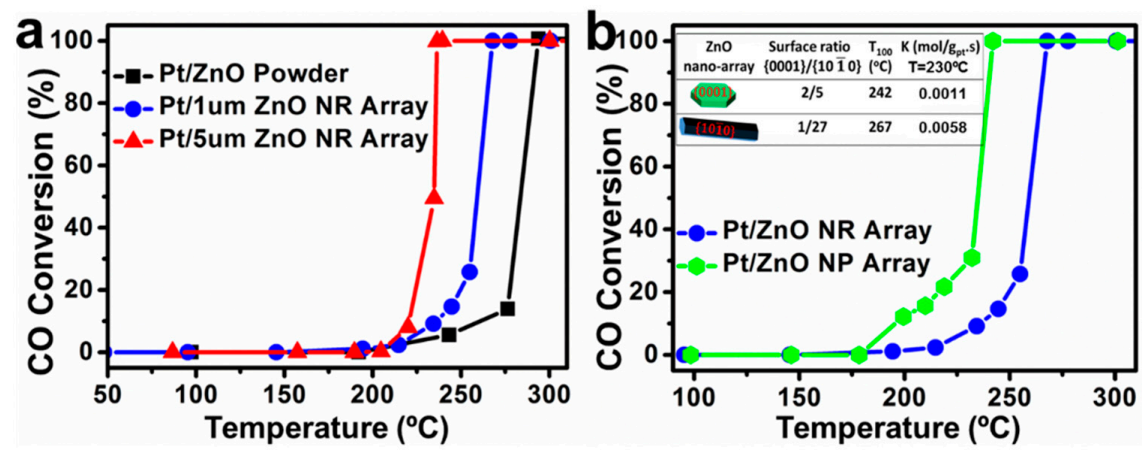

Figure 8. Catalytic $\mathrm{CO}$ oxidation performance of $\mathrm{Pt}$ loaded on $\mathrm{ZnO}$ nano-arrays with different lengths and shapes. (a) CO oxidation conversion as a function of temperature over $1 \mu \mathrm{m}$ and $5 \mu \mathrm{m}$ long Pt-loaded $\mathrm{ZnO}$ nanorod array and washcoated $\mathrm{Pt} / \mathrm{ZnO}$ powder catalysts. (b) CO oxidation conversion as a function of temperature over Pt-ZnO nanorod array and $\mathrm{Pt}-\mathrm{ZnO}$ nanoplate array. Reprinted with permission from [36], Copyright Elsevier, 2013. 
Figure 9a-i represents the different morphologies of manganese oxide nano-arrays grown on cordierite monoliths using hydrothermal method by controlling the recipes [25]. Three types of potassium salt oxidants with different redox potentials were used for the synthesis, i.e., $\mathrm{K}_{2} \mathrm{Cr}_{2} \mathrm{O}_{7}$, $\mathrm{KClO}_{3}$, and $\mathrm{K}_{2} \mathrm{~S}_{2} \mathrm{O}_{8}$, denoted as HM-DCM, HM-PCR, and HM-PSF, respectively. The different redox potentials of the manganese source $\left(\mathrm{Mn}^{2+}\right)$ and oxidants result in the formation of manganese oxide nano-arrays with different morphologies, surface area, and $\mathrm{CO}$ oxidation activities of different samples are shown in Figure 9j-m. Sharp and long nanowires can be obtained from $\mathrm{K}_{2} \mathrm{Cr}_{2} \mathrm{O}_{7}$ and $\mathrm{KClO}_{3}$ with slow growth rates due to the low reduction potentials, while shorter nanorods nano-arrays were obtained when $\mathrm{K}_{2} \mathrm{~S}_{2} \mathrm{O}_{8}$ was presented, but displayed an efficient $90 \% \mathrm{CO}$ conversion at $200{ }^{\circ} \mathrm{C}$ $\left(\mathrm{T}_{90}\right)$ without noble-metal loading. Reducibility tests for the three monolithic catalysts by hydrogen temperature-programmed reduction $\left(\mathrm{H}_{2}-\mathrm{TPR}\right)$ revealed an activation energy order of HM-PSF > HM-DCM > HM-PCR for CO oxidation. The characterizations of oxygen temperature-programmed desorption $\left(\mathrm{O}_{2}-\mathrm{TPD}\right)$ and XPS indicated the abundant surface-adsorbed oxygen and lattice oxygen, which contributed to the superior reactivity of HM-PSF. The straightforward synthetic process showed a scalable, low-cost, and template-free method to fabricate manganese oxide nano-array monolithic catalysts for exhaust treatment.
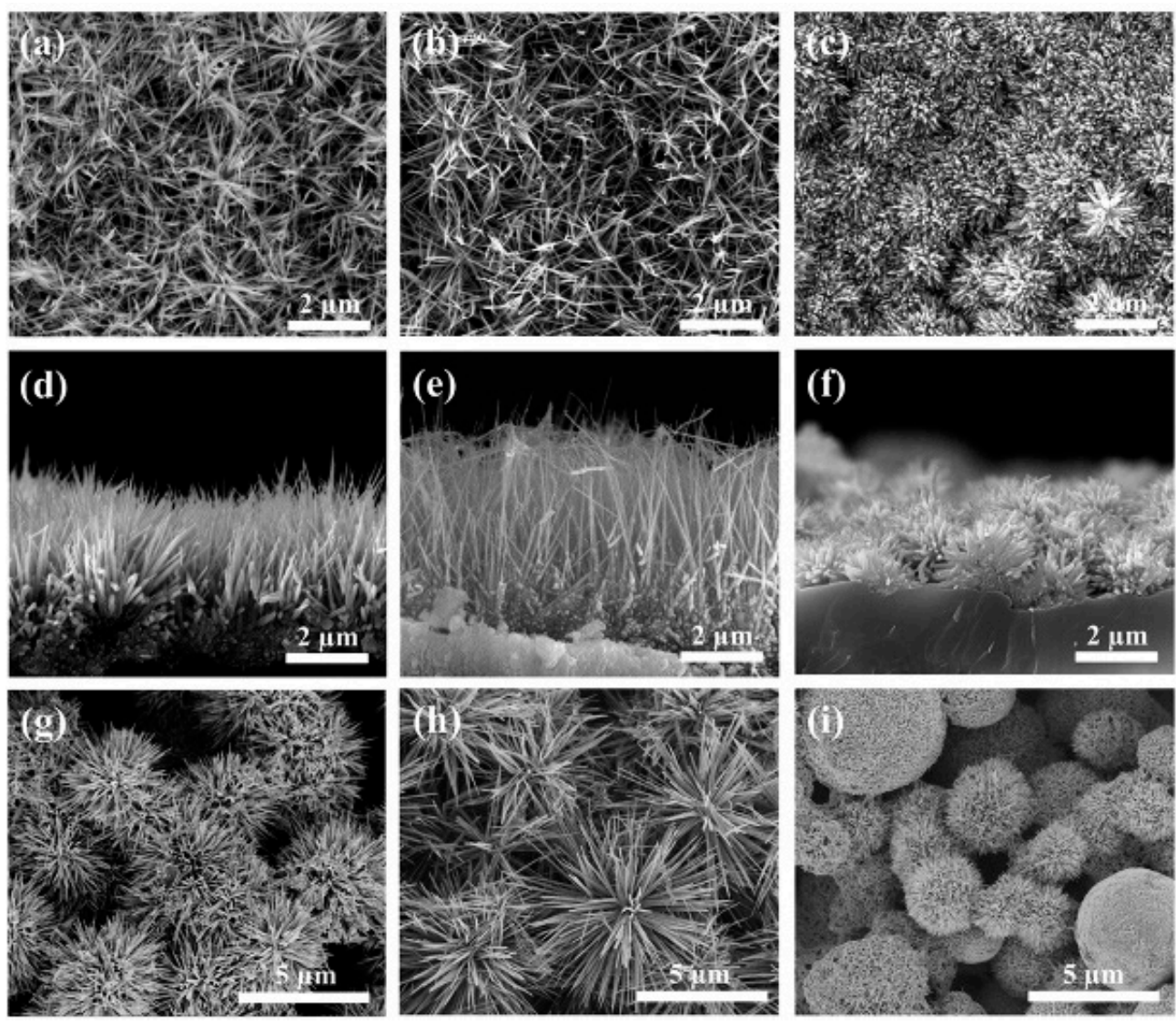

Figure 9. Cont. 
(j)

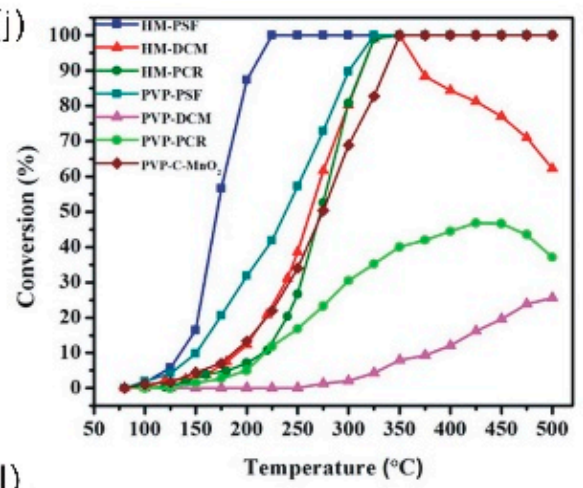

(I)

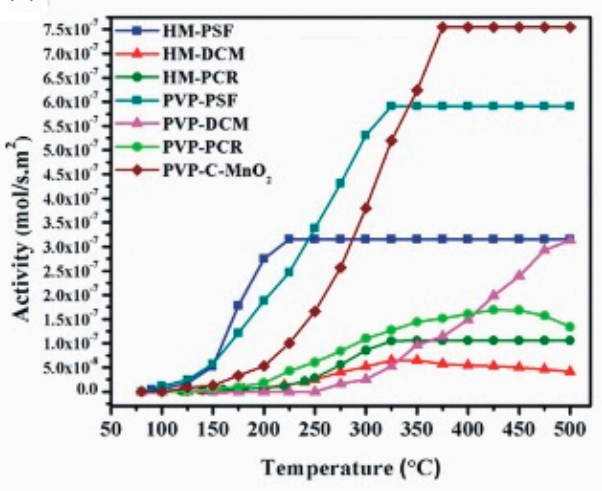

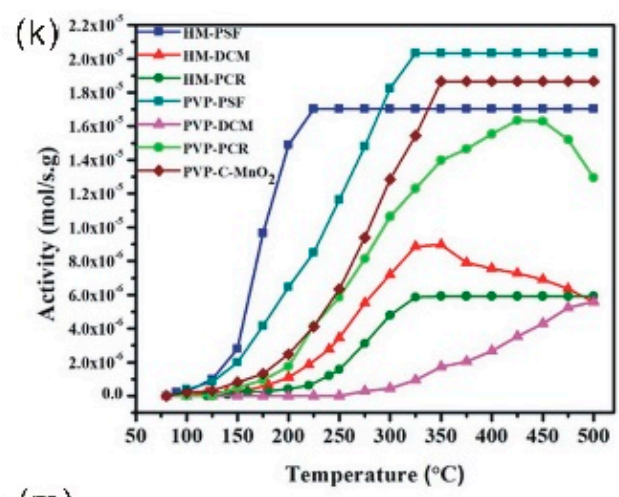

(m)

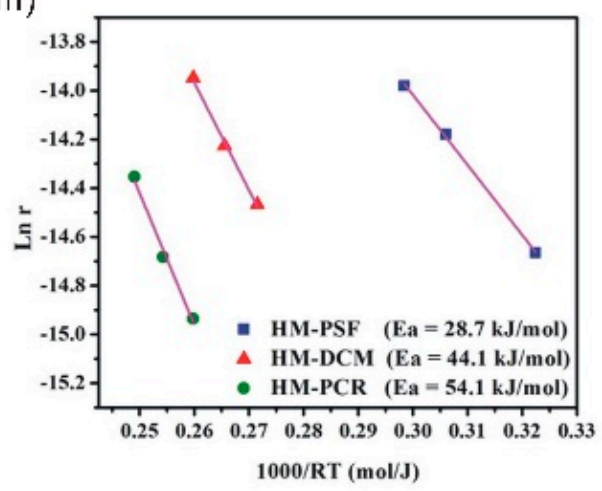

Figure 9. SEM images of manganese oxide nanorod arrays on the cordierite substrate: (a) top view and (d) cross-sectional view of $\mathrm{K}_{2} \mathrm{Cr}_{2} \mathrm{O}_{7}$ (HM-DCM); (b) top view and (e) cross-sectional view of $\mathrm{KClO}_{3}$ (HM-PCR); (c) top view and (f) cross-sectional view of $\mathrm{K}_{2} \mathrm{~S}_{2} \mathrm{O}_{8}$ (HM-PSF). SEM images of as-synthesized manganese oxide powders collected from each reaction: (g) HM-DCM; (h) HM-PCR; (i) HM-PSF; (j) Catalytic performance of manganese oxide nano-array-based and dip-coated monolithic catalysts for CO oxidation; (k) Calculated activities as a function of the temperature; (1) Calculated activities normalized by the surface area as a function of the temperature; (m) Arrhenius plot of the reaction kinetics and calculated activation energies. Reprinted with permission from [25], Copyright ACS, 2016.

\subsection{Guest Atoms and Defects Manipulation}

In addition to the manipulation of geometry shapes and enclosed crystal planes, the catalytic activity of nano-array-based monolithic catalysts could also be modified by introducing guest atoms and defects $[17,18]$. As an example, $\mathrm{Ni}$ was doped into the $\mathrm{Co}_{3} \mathrm{O}_{4}$ lattice to enhance the catalytic activity for methane and propane oxidation. The SEM images in Figure 10a,b displayed the morphology and orientation of the Ni-doped and pristine $\mathrm{Co}_{3} \mathrm{O}_{4}$ nanowires on the monolithic cordierite honeycombs. No phase segregation was observed when $\mathrm{Ni}$ was added as a dopant and the hierarchical nano-arrays retained their morphology. The corresponding HRTEM images in Figure 10c,d revealed the crystalline nature of the nanowires. Figure 10e,f shows the catalytic methane [18] and propane [17] oxidation performance of $\mathrm{Ni}_{\mathrm{x}} \mathrm{Co}_{3-\mathrm{x}} \mathrm{O}_{4}$ nano-arrays with controlled Ni concentration. The $\mathrm{Co}_{3} \mathrm{O}_{4}$ nano-arrays doped with $\mathrm{Ni}$ exhibited better methane and propane oxidation activity at low temperatures than the pristine $\mathrm{Co}_{3} \mathrm{O}_{4}$ nano-arrays, and could achieve complete $\mathrm{CH}_{4}$ and $\mathrm{C}_{3} \mathrm{H}_{8}$ combustion below $600{ }^{\circ} \mathrm{C}$ and $400{ }^{\circ} \mathrm{C}$, respectively. With the increased $\mathrm{Ni}$ doping concentration, reaction kinetics can be further promoted and complete $\mathrm{CH}_{4}$ and $\mathrm{C}_{3} \mathrm{H}_{8}$ conversion can be achieved at lower temperatures. According to the results of Density Functional Theory (DFT) calculations [17] on the dominant exposed \{110\} planes of the porous $\mathrm{M}_{0.5} \mathrm{Co}_{2.5} \mathrm{O}_{4}(\mathrm{M}=\mathrm{Co}, \mathrm{Ni})$ nanowires and Raman spectroscopy analysis, $\mathrm{Ni}$ atoms took the octahedral $\mathrm{Co}$ sites $\left(\mathrm{Co}^{3+}\right)$ in the spinel lattice, providing an active site for $-\mathrm{CH}_{3}$ adsorption to facilitate the oxidation. Moreover, the occupation of octahedral Co sites by Ni also led to the loss of positive charges. Such charge loss was compensated by the formation of oxygen vacancy, in order 
to maintain the charge neutrality. XPS spectra in Figure 10c revealed the deconvoluted O1s signals and indicated the increase of defective oxygen population as the Ni concentration was increased. It is believed that the rich surface oxygen defects can enhance the mobility of surface lattice oxygen species, which further promotes the $\mathrm{C}-\mathrm{H}$ activation and reaction kinetics.
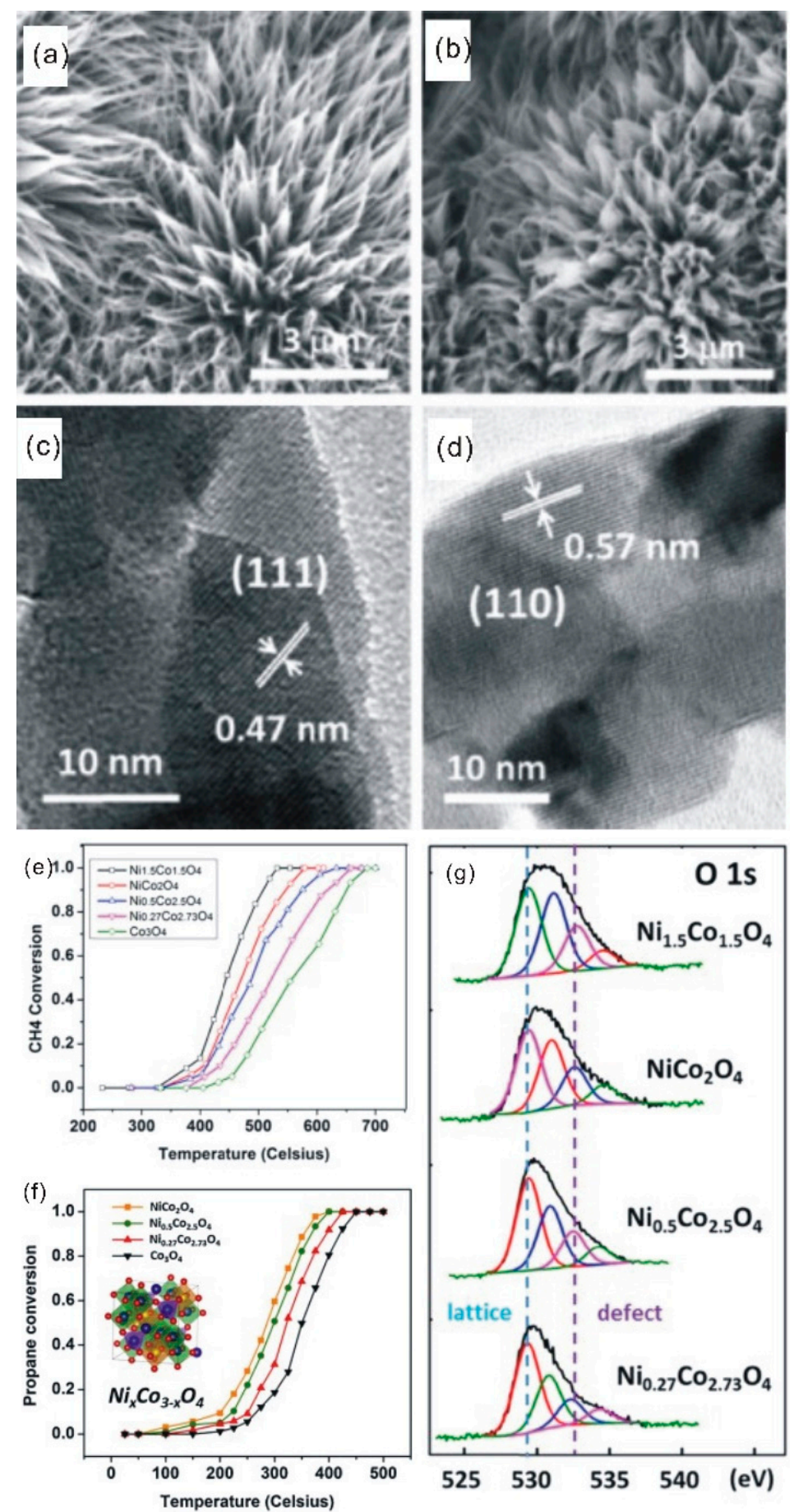

Figure 10. SEM images of the as-prepared nanowire array catalysts: (a) $\mathrm{Co}_{3} \mathrm{O}_{4}$ nano-arrays; (b) $\mathrm{Ni}$ doped $\mathrm{Co}_{3} \mathrm{O}_{4}$ nano-arrays $\left(\mathrm{Ni}_{0.27} \mathrm{Co}_{2.73} \mathrm{O}_{4}\right)$. High resolution TEM characterization of (c) $\mathrm{Co}_{3} \mathrm{O}_{4}$ nanowires; (d) $\mathrm{Ni}_{0.27} \mathrm{Co}_{2.73} \mathrm{O}_{4}$ nanowires. Catalytic (e) methane and (f) propane oxidation performance of Ni doped $\mathrm{Co}_{3} \mathrm{O}_{4}$ nano-arrays; (g) Deconvoluted XPS spectra of O1s for Ni doped $\mathrm{Co}_{3} \mathrm{O}_{4}$ with different Ni concentrations. Reprinted with permission from [17], Copyright Wiley, 2014. Reprinted with permission from [18], Copyright Elsevier, 2016. 


\subsection{Composite Nano-Array Catalysts}

Besides the Pt-supported metal oxides, metal oxide/perovskite nano-array-based composite catalysts were also fabricated and their activities were tested. In our previous work, $\mathrm{TiO}_{2} /(\mathrm{La}, \mathrm{Sr}) \mathrm{MnO}_{3}$ $\left(\mathrm{TiO}_{2} / \mathrm{LSMO}\right)$ composite nano-arrays on the planar substrates were successfully synthesized by combined hydrothermal growth and magnetron sputtering [26]. As shown in Figure 11a, $\mathrm{TiO}_{2} /$ LSMO nanorods with aligned and densely packed morphology vertically grew on the substrate. From Figure $11 \mathrm{~b}$, the length of the composite nanorods was identified as $\sim 1 \mu \mathrm{m}$ and the diameter was $\sim 30-100 \mathrm{~nm}$. The as-prepared composites were composed of single crystalline $\mathrm{TiO}_{2}$ nanorod core with amorphous perovskite shell, which could be turned into crystalline after thermal annealing at $800{ }^{\circ} \mathrm{C}$ for $3 \mathrm{~h}$. As shown in Figure 11c, after annealing, the typical single $\mathrm{TiO}_{2} / \mathrm{LSMO}$ composite nanorod with a ridged LSMO shell could be clear identified. $\mathrm{TiO}_{2} / \mathrm{LSMO}$ core-shell nanorod array achieved $100 \% \mathrm{CO}$ conversion at $400{ }^{\circ} \mathrm{C}$, while the highest $\mathrm{CO}$ conversion efficiency of pure $\mathrm{TiO}_{2}$ nanorod array was only $20 \%$ at $450{ }^{\circ} \mathrm{C}$. The lower $100 \%$ CO conversion temperature compared to the reported powder form LSMO catalysts could be attributed to the high surface area enabled by the nano-array configuration and the oxygen vacancies of LSMO film.
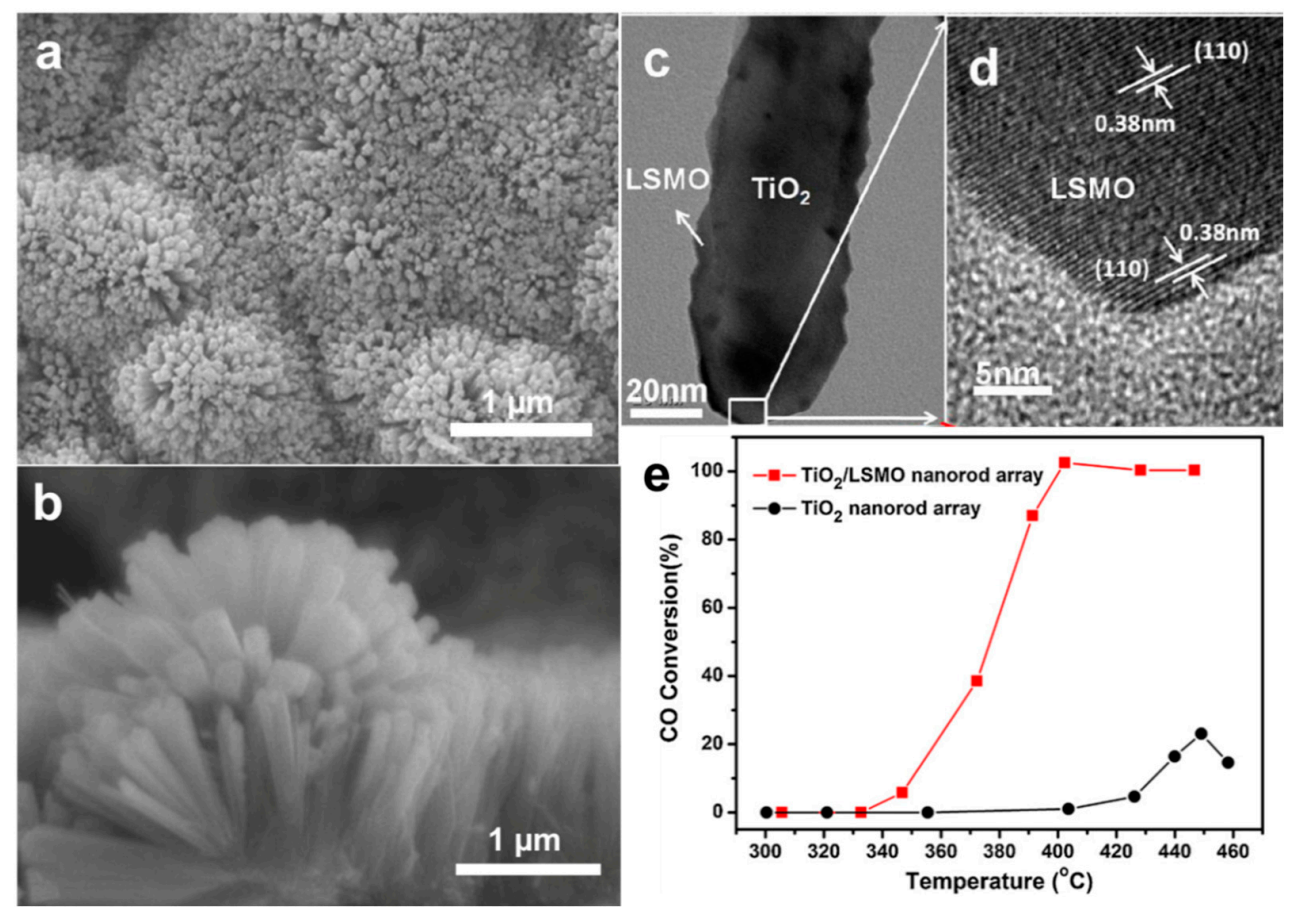

Figure 11. (a) A top view and (b) SEM cross-sectional view image of $\mathrm{TiO}_{2} / \mathrm{LSMO}$ nanorod arrays on $\mathrm{Si}$ substrates; (c) Bright field and (d) HRTEM images of a typical $\mathrm{TiO}_{2} / \mathrm{LSMO}$ core-shell nanorod after $800{ }^{\circ} \mathrm{C}$ annealing; (e) Catalytic $\mathrm{CO}$ oxidation performance of $\mathrm{TiO}_{2} / \mathrm{LSMO}$ core-shell nanorod arrays and $\mathrm{TiO}_{2}$ nanorod arrays. Reprinted with permission from [26], Copyright Elsevier, 2012.

In addition to the $\mathrm{TiO}_{2} /$ LSMO composite nanorod catalysts, we have also developed $\mathrm{ZnO} /$ perovskite $\left(\mathrm{LaBO}_{3}, \mathrm{~B}=\mathrm{Mn}, \mathrm{Co}\right.$, and $\mathrm{Ni}$, noted as $\mathrm{LMO}, \mathrm{LCO}$, and $\mathrm{LNO}$, respectively) core-shell nanorod arrays within $3 \mathrm{D}$ cordierite substrates by combining hydrothermal strategy with colloidal deposition synthesis. The cross-section views of $\mathrm{ZnO}$ nanorod arrays rooted on monolithic substrate, and the derived $\mathrm{ZnO} / \mathrm{LMO}, \mathrm{ZnO} / \mathrm{LCO}$ and $\mathrm{ZnO} / \mathrm{LNO}$ core-shell nanorod arrays are shown in Figure 12a-d. Figure 12e,f displayed the bright field TEM and HRTEM images of $\mathrm{ZnO} / \mathrm{LCO}$ core-shell nanorods. Propane oxidation was performed to evaluate the oxidation activities of these catalysts. As shown in Figure 12g,h, the $\mathrm{ZnO}$ /perovskite composite catalysts demonstrated much lower light-off temperatures than the pure $\mathrm{ZnO}$ nano-array catalysts. Comparing the three types of $\mathrm{ZnO} /$ perovskite 
nano-array based catalysts, the $\mathrm{ZnO} / \mathrm{LCO}$ catalyst showed the best catalytic activity at relatively low temperatures, whereas $\mathrm{ZnO} / \mathrm{LNO}$ displayed highest propane conversion at about $650{ }^{\circ} \mathrm{C}$.
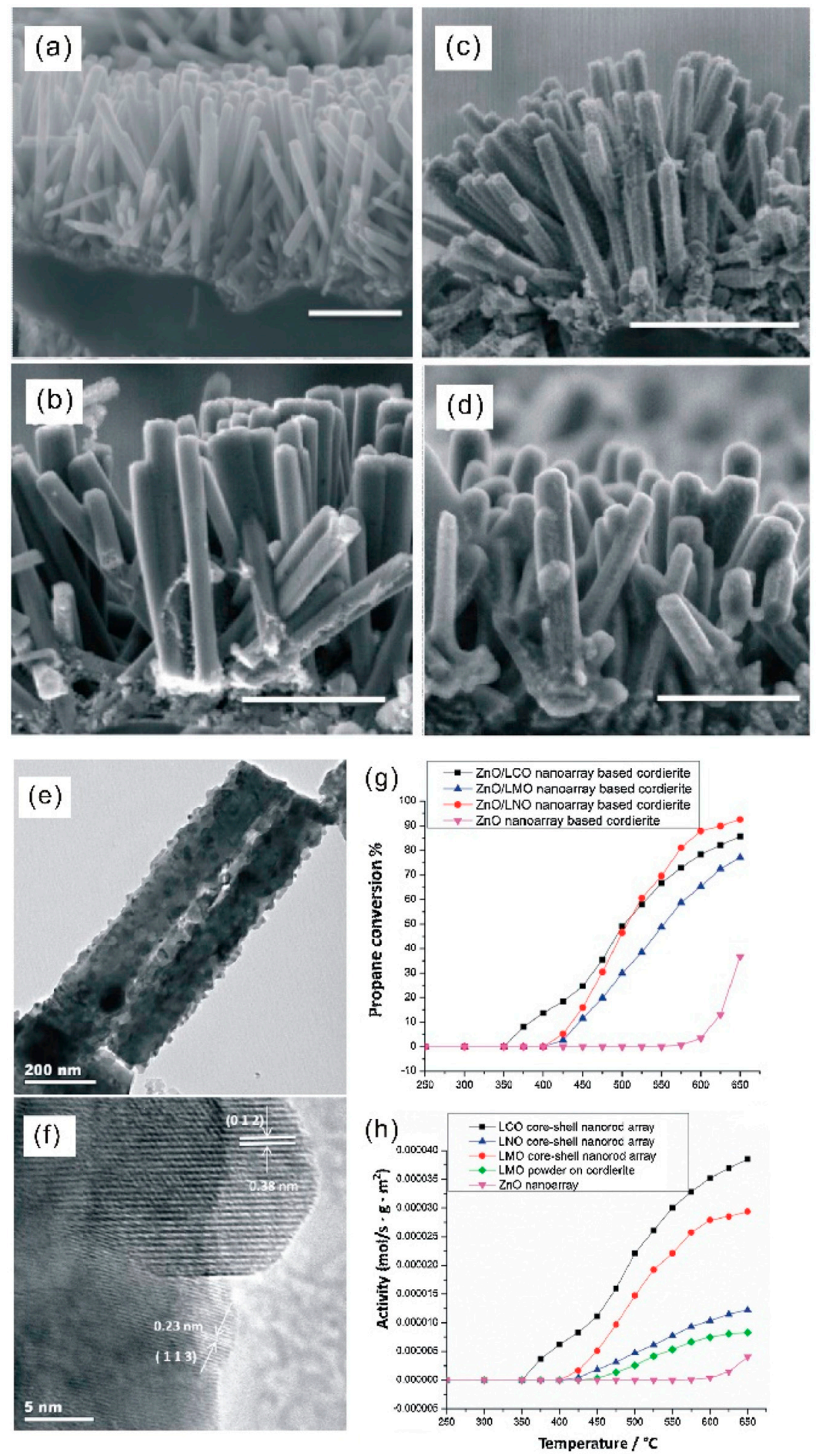

Figure 12. SEM cross-section view of (a) $\mathrm{ZnO}$ nanorod arrays rooted on monolithic cordierite substrate; (b) $\mathrm{ZnO} / \mathrm{LMO}$; (c) $\mathrm{ZnO} / \mathrm{LCO}$; and (d) $\mathrm{ZnO} / \mathrm{LNO}$ core-shell nanorod array catalysts. Scale bars in SEM images are $1 \mu \mathrm{m}$. (e) Bright field TEM and (f) HRTEM images of ZnO/LCO core-shell nanorods; (g) Propane oxidation over $\mathrm{ZnO}$ and $\mathrm{ZnO} /$ perovskite nano-array catalysts; (h) Activity of perovskite catalysts shown as a function of temperature. Reprinted with permission from [15], Copyright Elsevier, 2015. 


\section{Stability Properties}

Wash-coated catalysts exhibit layer-type structure formed by the several deposition procedures, including the steps of dip-coating or impregnation, drying, calcination, and the multi-repeated times before achieving the desired coating amount. Because of the different interfaces within the wash-coated structure and larger wash-coated particles compared with substrate pores, the adhesion and cohesion properties of the wash-coat catalysts were serious issues for the performance stability, resulting in degradation of catalyst efficiency once active catalysts being removed.

The mechanical stability of wash-coated catalysts involved a combination of adhesion and cohesion, while the failure of active components included adhesive and cohesive failure, which occurred at the washcoat layer-cordierite substrate interface and within the washcoat layer, correspondingly [27]. It was reported that the pretreating agent, solid content, additive, amount of additive, and wash-coating times, had great effects on the loading and on the properties of adhesion and cohesion for washcoats [28]. Recent studies through the combined Focused Ion Beam-Scanning Transmission Electron Microscope (FIB-STEM) characterization technology revealed that the spatial distribution of the different constituents of multicomponent catalysts on the monolithic substrates were significantly influenced by the wash-coating processes, independent of either the nature of the support (ceramic or metallic) or the total amount of deposited material. An interaction between deposition components may be much more limited than that in the initial powders. It was necessary to perform an assessment of the deposited catalysts layer after wash-coat procedures [29,30].

The performance of stability with respect to thermal stability, hydrothermal stability, and mechanical stability was crucial to catalytic processes at high temperatures, which are even more harsh and complex in practical working conditions [31,32]. Therefore, it is essential to conduct a series of experiments to evaluate the evolution of catalysts during different processes.

Currently, nano-array structured catalysts have exhibited exciting advantageous features on the thermal stability and mechanical robustness over the conventional wash-coated catalysts. Guo et al. [9] synthesized a series of nano-array $\mathrm{ZnO}, \mathrm{TiO}_{2}$, and $\mathrm{Co}_{3} \mathrm{O}_{4}$ on cordierite monoliths. From the results of thermogravimetric analysis (TGA) and differential scanning calorimetry (DSC) tests up to $1000{ }^{\circ} \mathrm{C}$, as shown in Figure 13, $\mathrm{TiO}_{2}$ and $\mathrm{ZnO}$ nanorods possessed a very stable structure with only less than $0.5 \%$ mass loss, while $\mathrm{Co}_{3} \mathrm{O}_{4}$ exhibited $\sim 2.5 \%$ mass loss. After an isothermal annealing process under $800{ }^{\circ} \mathrm{C} / 100 \mathrm{~h}$, all nano-array structured catalysts exhibited less than $1 \%$ mass change without phase transformation. For instance, the structure of as-prepared porous $\mathrm{Co}_{3} \mathrm{O}_{4}$ nanowires only changed within the first $24 \mathrm{~h}$ at $800{ }^{\circ} \mathrm{C}$, being retained further during the rest of the isothermal process. Within the first $24 \mathrm{~h}$, the relatively smooth surfaces turned into zigzag periodic and rough nanowires, the pore volume dropped by $\sim 46 \%$, and the Brunauer-Emmett-Teller (BET) surface area decreased from $8 \mathrm{~m}^{2} / \mathrm{g}$ to $4 \mathrm{~m}^{2} / \mathrm{g}$. Compared to the surface area decrease in conventional powder-type catalysts, $\mathrm{ZnO}, \mathrm{TiO}_{2}$, and $\mathrm{Co}_{3} \mathrm{O}_{4}$ nano-array catalysts showed much higher thermal stability [33-35].

To identify the stability of nano-array structures in reductive atmosphere, temperature-programmed $\mathrm{H}_{2}$ reduction (TPR- $\mathrm{H}_{2}$ ) was tested. As-prepared nano-array structured catalysts exhibited good thermal stability in reductive atmosphere as well. $\mathrm{TiO}_{2}$ nanorods on cordierite monolith showed high stability and inertness in $\mathrm{H}_{2}$ atmosphere without structure changes up to $750{ }^{\circ} \mathrm{C}$, while $\mathrm{ZnO}$ nanorods stayed stable in reduced atmosphere below $450{ }^{\circ} \mathrm{C}$ [36].

High velocity air flow was utilized to simulate the practical working conditions for vehicle aftertreatment system. All the as-prepared nanostructure exhibited high stability and maintained the array structure and morphology either after 200 cycles of high pulsatile air flow (switched between 0 and $60 \mathrm{~L} / \mathrm{min}$ ) or under the static air with $50 \mathrm{~L} / \mathrm{min}$ flux for 10 days. Additionally, directly grown single crystal $\mathrm{TiO}_{2}$ and $\mathrm{ZnO}$ nano-array monolithic catalysts showed great stability and vibration resistance to mechanical vibrations $(>1 \mathrm{~h})$ introduced by Sonicator $(42 \mathrm{KHz}, 135 \mathrm{~W})$ in both distilled water and ethanol [36]. 

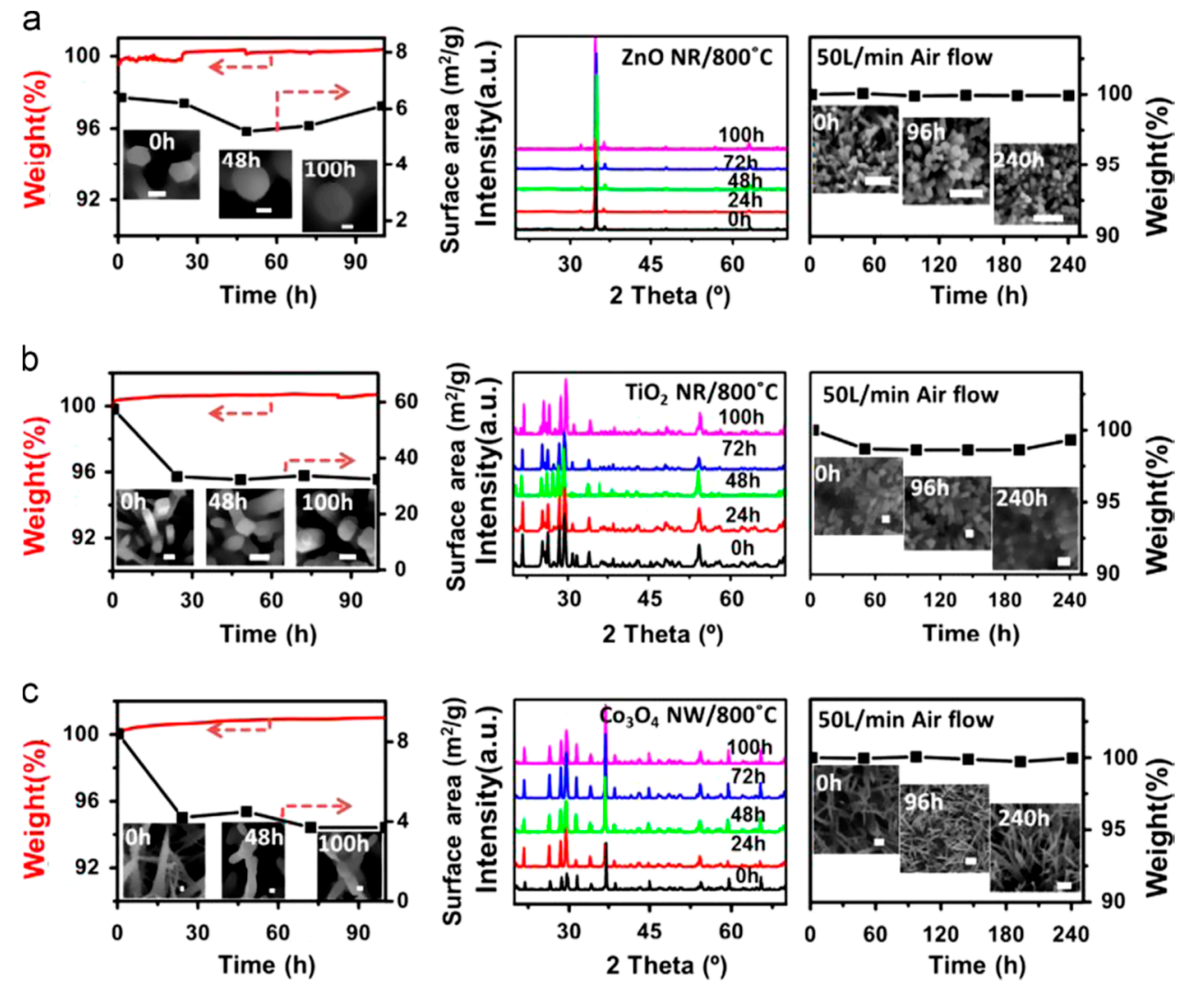

Figure 13. Thermal and mechanical robustness of metal oxide nano-array based monolithic catalysts in ambient atmosphere. (a) 1- $\mu \mathrm{m} \mathrm{ZnO}$ nanorod array grown on stainless steel monolith; (b) 1- $\mu \mathrm{m}$ $\mathrm{TiO}_{2}$ nanorod array grown on cordierite monolith; (c) 8- $\mu \mathrm{m} \mathrm{Co}_{3} \mathrm{O}_{4}$ porous nanowire array grown on cordierite monolith. Reprinted with permission from [36], Copyright Elsevier, 2013.

Besides thermal and mechanical stability, long-term stability of catalytic performance is a substantial important factor for a reliable catalyst. Ren et al. [7] reported that the as-prepared $\mathrm{Co}_{3} \mathrm{O}_{4}$ nano-array catalysts by three different $C o$ precursors exhibited remarkable catalytic stability during the cyclic NO oxidation test, as shown in Figure 14, which was comparable to PGM catalysts. The testing conditions were 10 catalytic cycles with temperature ramping from $25^{\circ} \mathrm{C}$ to $400{ }^{\circ} \mathrm{C}$. Compared with the freshly prepared honeycombs, all catalytic honeycombs experienced less than $5 \%$ degradation after 5 cycles. The weight of each $\mathrm{Co}_{3} \mathrm{O}_{4}$ nano-array catalyst stayed constant, which indicated no catalyst loss throughout the test.

Repeated heating and cooling is inevitable for catalysts in the automotive application. Ren et al. [16] investigated the stability of nano-array-based catalysts by mimicking the temperature fluctuation by cyclic temperature within $150-400{ }^{\circ} \mathrm{C}$ (Figure 15). The maximum NO conversion temperature stayed the same during cooling process and no degradation of catalytic activity was observed. At the temperature where the maximum conversion was achieved, a stable isothermal catalytic performance could be obviously demonstrated.

In Figure 16, the stability of Ni-doped $\mathrm{Co}_{3} \mathrm{O}_{4}$ catalytic activity was characterized by controlling the reaction temperatures at which $10 \%$ and $90 \%$ conversion of methane were achieved according to the light-off curve [17]. Ni substitution was proved to enhance both activity and stability of the catalyst towards methane combustion. Another isothermal stability test performed at $600{ }^{\circ} \mathrm{C}$ showed the methane conversion decayed faster in the case of $\mathrm{Ni}_{0.5} \mathrm{Co}_{2.5} \mathrm{O}_{4}$ nano-arrays compared with that of $\mathrm{Co}_{3} \mathrm{O}_{4}$ nano-arrays. Higher $\mathrm{Ni}$-doped $\mathrm{Co}_{3} \mathrm{O}_{4}$ catalyst, $\mathrm{NiCo}_{2} \mathrm{O}_{4}$, was found to decompose at temperature higher than $400{ }^{\circ} \mathrm{C}$, producing a small amount of $\mathrm{NiO}$ precipitation and $\mathrm{Ni}_{\mathrm{x}} \mathrm{Co}_{3-\mathrm{x}} \mathrm{O}_{4}$ matrix. 

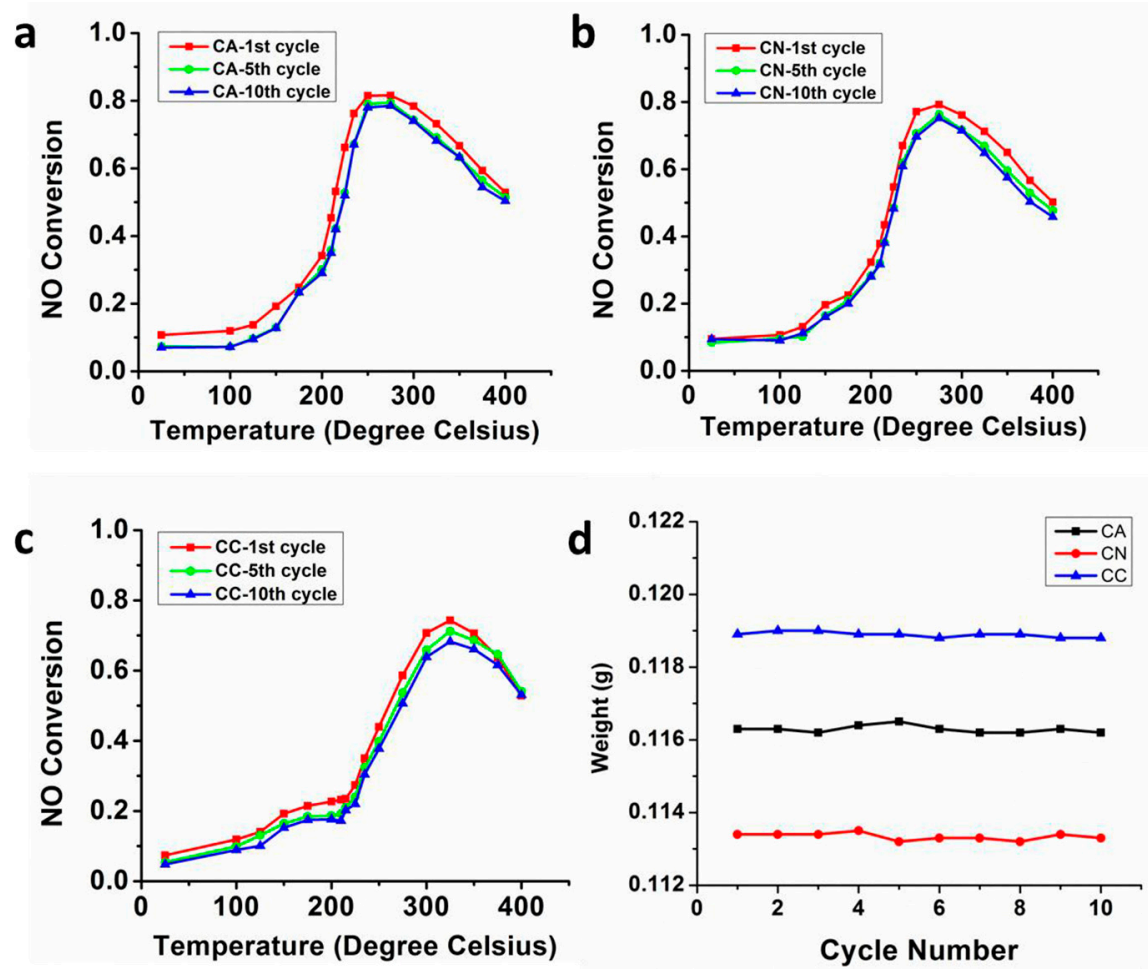

Figure 14. Cyclic catalytic NO oxidation performance by (a) CA; (b) $\mathrm{CN}$ and (c) CC; (d) Weight measurement of catalytic honeycombs during cyclic test. Reprinted with permission from [16], Copyright RSC, 2013.

a

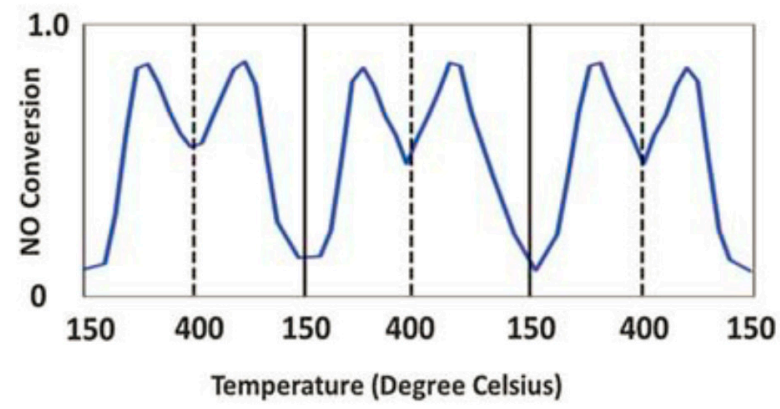

b

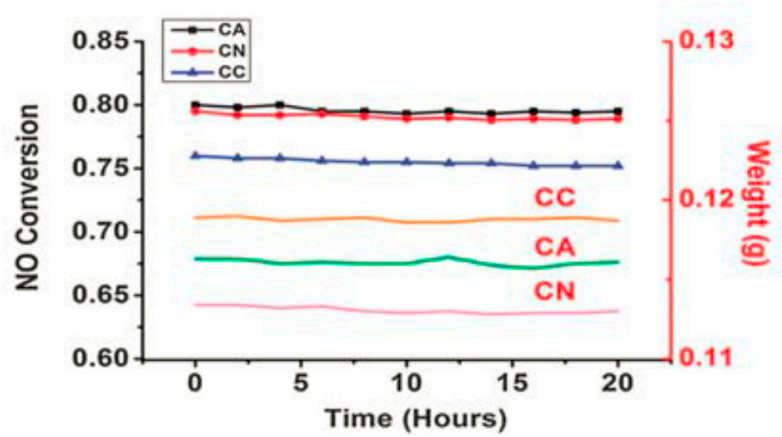

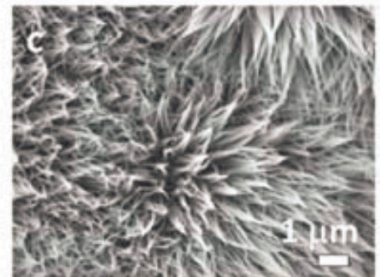
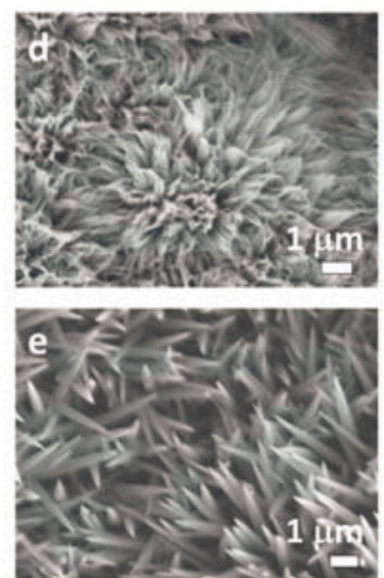

Figure 15. (a) Catalytic NO oxidation performance with repeated heating and cooling; (b) NO oxidation efficiency and weight measurement during long term steady investigation at $275^{\circ} \mathrm{C}$ for $\mathrm{CA}$ and $\mathrm{CN}$ and $325^{\circ} \mathrm{C}$ for CC; (c-e) SEM images of CN, CA and CC respectively after all stability tests. Reprinted with permission from [16], Copyright RSC, 2013. 

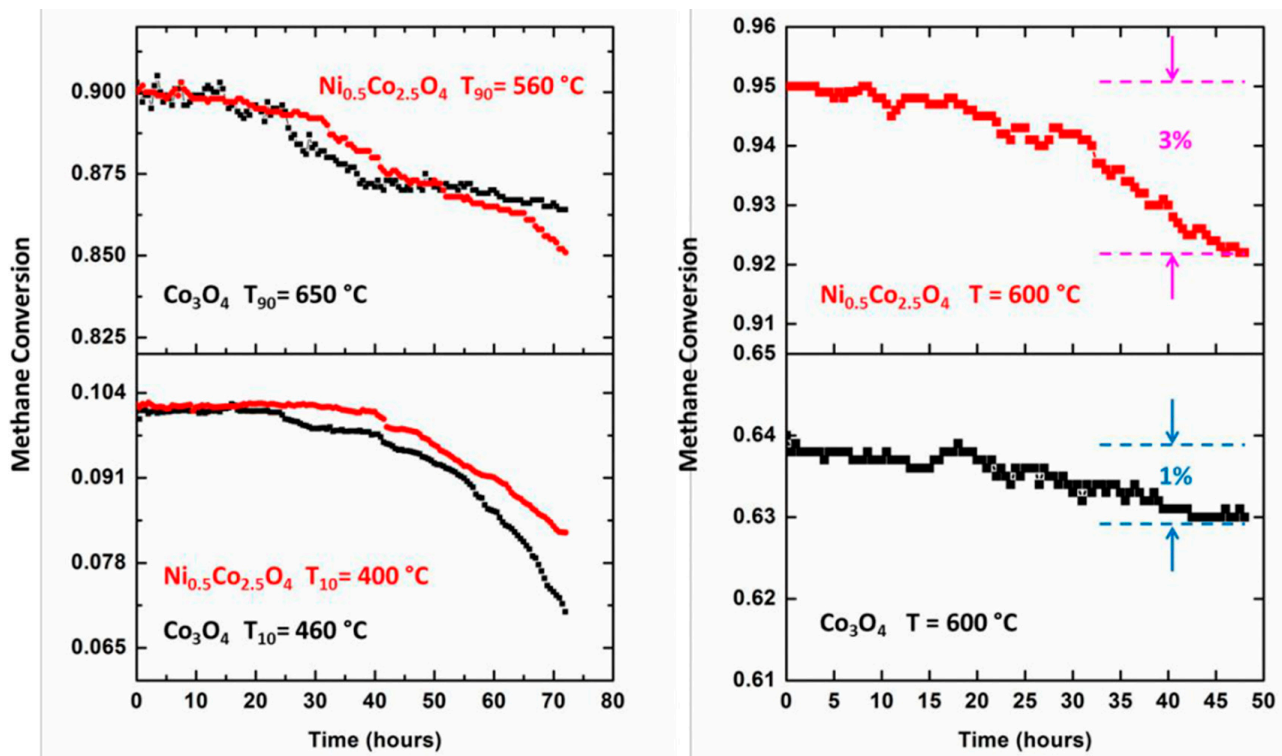

Figure 16. Catalytic stability test results of $\mathrm{Ni}_{0.5} \mathrm{Co}_{2.5} \mathrm{O}_{4}$ and $\mathrm{Co}_{3} \mathrm{O}_{4}$ nano-arrays for methane combustion. Reprinted with permission from [17], Copyright Wiley, 2014.

In practical applications, surface coating layers are inevitably exposed to the environment of either liquid water or steam. Water/steam sometimes could seriously undermine the catalyst performance. In Figure 17, the hydrothermal stability of $\mathrm{TiO}_{2}$ nanorod arrays coated hybrid monolith substrates at extreme conditions (100\% humidity) was evaluated [9]. After being aged in boiling de-ionized (DI) water at $120{ }^{\circ} \mathrm{C}$ for $24 \mathrm{~h}$, the morphology of $\mathrm{TiO}_{2}$ nanorod arrays kept densely packed and uniform distribution without crystal transformation for the single crystal brookite structure. Similar to $\mathrm{ZnO}-$ based nano-array catalysts, the array structure and surface morphology of $\mathrm{ZnO}$ retained very well without visible changes.
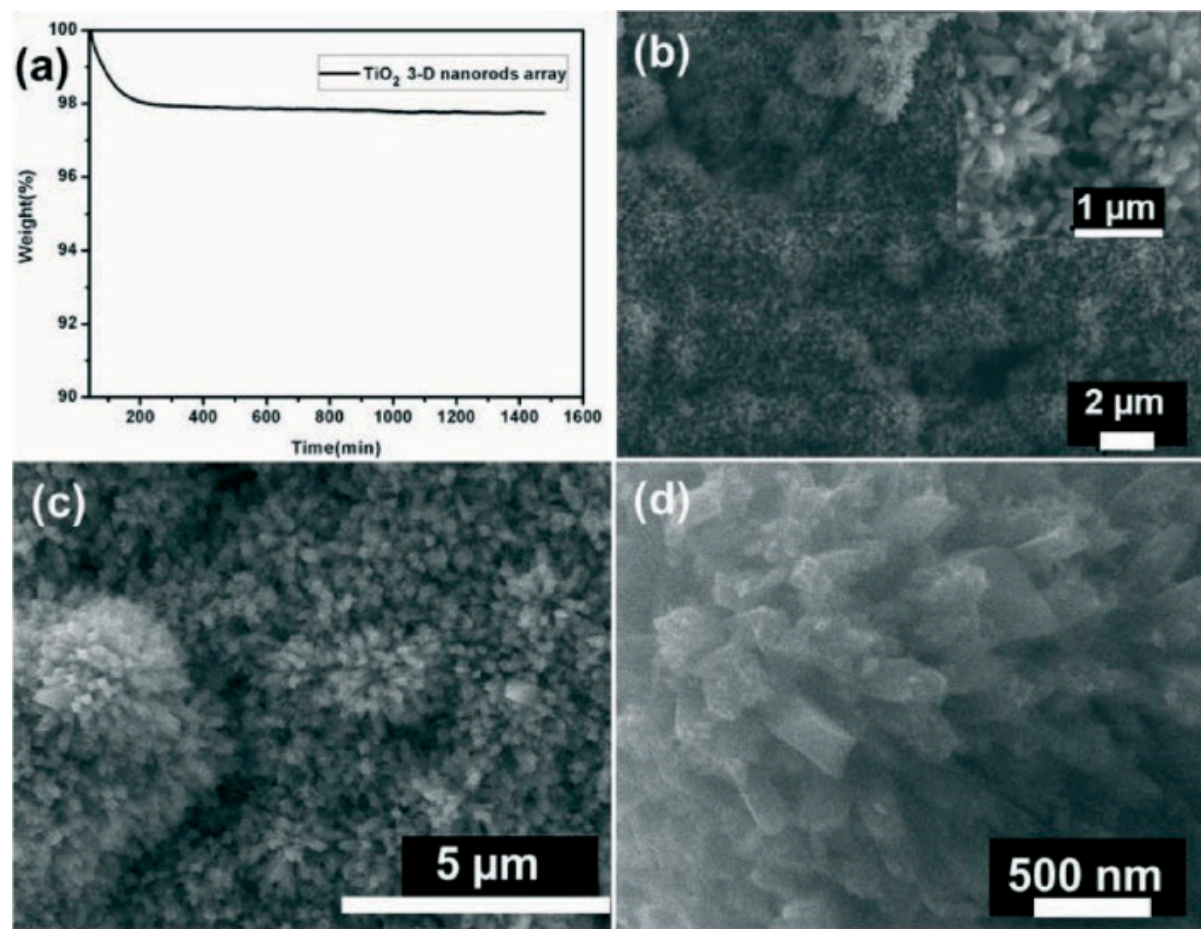

Figure 17. Cont. 


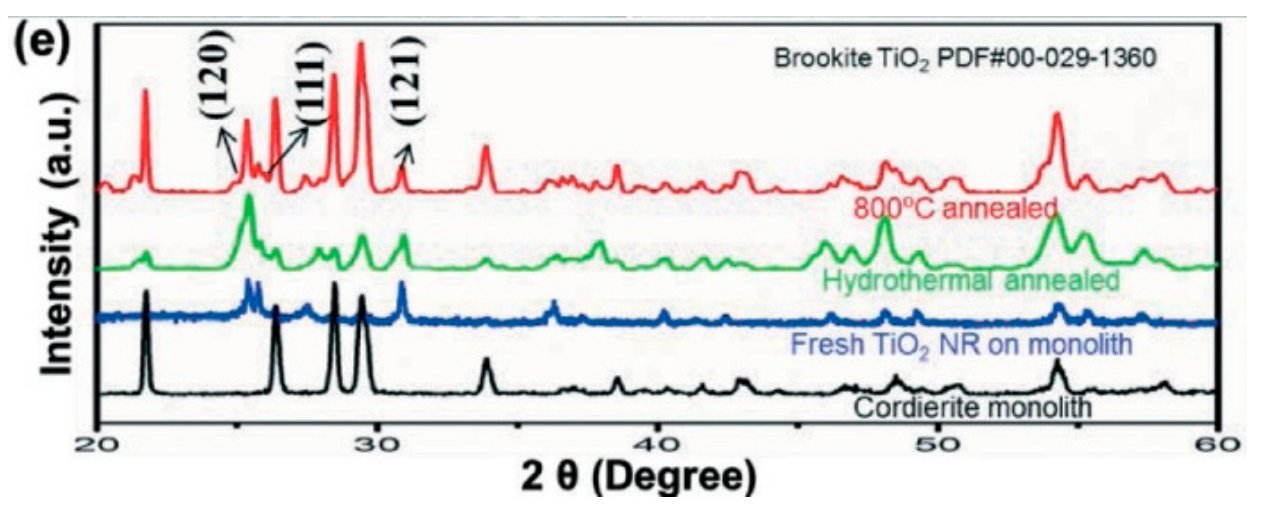

Figure 17. (a) Mass loss vs. time spectra of $\mathrm{TiO}_{2}$ nanorods array annealed at $800{ }^{\circ} \mathrm{C} / 24 \mathrm{~h}$; (b) SEM image after $800{ }^{\circ} \mathrm{C} 24 \mathrm{~h}$ annealing; inset is the high magnification image; $\mathrm{TiO}_{2}$ nanorods array after aging in boiling DI water at $120^{\circ} \mathrm{C}$ for $24 \mathrm{~h}$ : SEM images of low magnification (c) and high magnification image (d); (e) XRD spectra of cordierite monolith, fresh $\mathrm{TiO}_{2}$ nanorod arrays on monolith, hydrothermally aged $\mathrm{TiO}_{2}$ nanorod arrays on monolith, and $800{ }^{\circ} \mathrm{C}$ annealed $\mathrm{TiO}_{2}$ nanorod arrays on monolith. Reprinted with permission from [9], Copyright RSC, 2013.

\section{Conclusions and Outlook}

In conclusion, this review summarized the recent progress on nano-array-based structured catalysts, demonstrating their advantages built upon and over the conventional wash-coated catalysts. The basic procedures for wash-coating process were briefly surveyed, summarizing the challenges facing the development of powder-wash-coated monolithic catalysts, including the various variables that are short of rational control during preparation, non-uniform distribution, and difficulties in correlation between their structure and performance, and therefore in rational design for desired properties. To address those issues and build upon the traditional washcoat monolithic catalysts, nano-array-based structured catalysts were invented and have been researched and developed lately, with various material systems successfully demonstrated including $\mathrm{Al}_{2} \mathrm{O}_{3}, \mathrm{CeO}_{2}, \mathrm{Co}_{3} \mathrm{O}_{4}, \mathrm{MnO}_{2}, \mathrm{TiO}_{2}$, $\mathrm{ZnO}, \mathrm{LaMnO}_{3}, \mathrm{LaCo}_{3}, \mathrm{LaNiO}$, and $\mathrm{LaFeO}_{3}$. The critical procedures that may be involved for the fabrication include (1) deposition of seeding layer; (2) in situ growth of nano-arrays; and (3) loading of catalytic materials.

By using hydrothermal synthesis facilitated by mechanical agitation such as magnetic stirring, and continuous flow process, not only the synthesis of small-size 3D integration of nano-arrays into the monolithic substrate was present, but also the scalable fabrication was demonstrated for industrially relevant manufacturing. After successful synthesis of the nano-array structured catalysts, the characterization and tests were conducted to confirm their excellent catalytic performance as compared with traditional PGM catalysts. As an important characteristic of the nano-array catalysts, performance tunability has been demonstrated by (1) morphology and geometry adjustment; (2) impurity and defects manipulation; and (3) composite nano-array catalyst manufacture. Finally, a series of stability tests under different conditions was used to demonstrate a superior stability again catalytic deactivation.

In summary, riding upon the conventional wash-coated monolithic catalysts, nano-array-based structured catalysts present a few critically promising aspects that could prove important for its potential practical implementation in our society in the near future:

(a) Cost-effective fabrication methods without multi-step complex processes and binders or additives involved;

(b) Well-defined structure with good uniformity on the monolithic substrates to control and predict the desired structure; 
(c) Capability to tune catalysts performance and potential to rationally design better catalysts with excellent stable properties.

As such, a number of benefits of nano-array-based monolithic catalysts have been proven with respect to the traditional wash-coated catalysts, such as excellent robustness, high material utilization efficiency, and excellent catalytic functions and functional stability, as well as inherent high-flux and low pressure-drop due to usually ultrathin micro-scale coating thickness in monolith channel surfaces. In the near future, a few important directions need to be investigated more intensively. For example, the scalable manufacturing of nanostructure arrays on product-relevant large monolithic substrates with good uniformity, high yield and throughput will be a critical step toward meeting industry practical applications. Therefore, a more in-depth and systematic study shall be critical toward scalable nanomanufacturing of nano-array integrated structured catalysts or reactors using both batch and continuous flow synthetic strategies. Such an interdisciplinary study involves various materials, mechanical, and chemical science, and related engineering such as materials engineering, chemical engineering, mechanical engineering, flow chemistry, as well as continuous flow reactor design and engineering. Another important direction is that the nano-array structured catalysts could be extended for applications in more catalytic reactions in both heterogeneous and homogeneous scenarios considering its intrinsic high-flux flow through reactor configuration could be rather beneficial, even the complex reactions under harsh environments. Meanwhile, in a nano-array-integrated flow-through system, the possible distinctions or advantages in mass transport and reaction kinetics are unclear with respect to the washcoat-based monolithic reactors, which present another interesting opportunity for further understanding and scientific advancement. Specifically, a mechanistic understanding could be very intriguing with respect to metal-oxide interfaces and gas-solid interaction in rather well-defined micro or nano-confined spaces in order to achieve the desired and predictive performance in the more defined chemical and physical reactor spaces.

Acknowledgments: The authors are grateful for the financial support from the US Department of Energy (Award Nos. DE-EE0006854 and DE-EE0000210) and the US National Science Foundation (Award No. CBET-1344970).

Author Contributions: P.-X.G., J.W., and X.L. perceived and conducted the review idea and outline, J.W, X.L., and P.-X.G. co-wrote and finalized the manuscript together.

Conflicts of Interest: The authors declare no conflict of interest.

\section{References}

1. Lundberg, B.; Sjöblom, J.; Johansson, Å.; Westerberg, B.; Creaser, D. Doc modeling combining kinetics and mass transfer using inert washcoat layers. Appl. Catal. B Environ. 2016, 191, 116-129. [CrossRef]

2. Rhys-Tyler, G.A.; Legassick, W.; Bell, M.C. The significance of vehicle emissions standards for levels of exhaust pollution from light vehicles in an urban area. Atmos. Environ. 2011, 45, 3286-3293. [CrossRef]

3. Bielaczyc, P.; Woodburn, J.; Szczotka, A. An assessment of regulated emissions and $\mathrm{CO}_{2}$ emissions from a European light-duty CNG-fueled vehicle in the context of Euro 6 emissions regulations. Appl. Energy 2014, 117, 134-141. [CrossRef]

4. Williams, J.L. Monolith structures, materials, properties and uses. Catal. Today 2001, 69, 3-9. [CrossRef]

5. Elmer, T.H. Ultra-Low Expansion Ceramic Articles. U.S. Patent 3958058 A, 18 May 1976.

6. Avila, P.; Montes, M.; Miró, E.E. Monolithic reactors for environmental applications: A review on preparation technologies. Chem. Eng. J. 2005, 109, 11-36. [CrossRef]

7. Nijhuis, T.A.; Beers, A.E.; Vergunst, T.; Hoek, I.; Kapteijn, F.; Moulijn, J.A. Preparation of monolithic catalysts. Catal. Rev. 2001, 43, 345-380. [CrossRef]

8. Ren, Z.; Guo, Y.; Liu, C.-H.; Gao, P.-X. Hierarchically nanostructured materials for sustainable environmental applications. Front. Chem. 2013, 1, 18. [CrossRef] [PubMed]

9. Guo, Y.; Liu, G.; Ren, Z.; Piyadasa, A.; Gao, P.-X. Single crystalline brookite titanium dioxide nanorod arrays rooted on ceramic monoliths: A hybrid nanocatalyst support with ultra-high surface area and thermal stability. CrystEngComm 2013, 15, 8345-8352. [CrossRef] 
10. Chen, H.; Rui, Z.; Ji, H. Monolith-like $\mathrm{TiO}_{2}$ nanotube array supported Pt catalyst for $\mathrm{HCHO}$ removal under mild conditions. Ind. Eng. Chem. Res. 2014, 53, 7629-7636. [CrossRef]

11. Ren, Z.; Guo, Y.; Gao, P.-X. Nano-array based monolithic catalysts: Concept, rational materials design and tunable catalytic performance. Catal. Today 2015, 258, 441-453. [CrossRef]

12. Wang, S.; Ren, Z.; Guo, Y.; Gao, P.-X. Nano-array integrated monolithic devices: Toward rational materials design and multi-functional performance by scalable nanostructures assembly. CrystEngComm 2016, 18, 2980-2993. [CrossRef]

13. Xu, S.; Wang, Z.L. One-dimensional zno nanostructures: Solution growth and functional properties. Nano Res. 2011, 4, 1013-1098. [CrossRef]

14. Xiao, W.; Guo, Y.; Ren, Z.; Wrobel, G.; Ren, Z.; Lu, T.; Gao, P.-X. Mechanical-agitation-assisted growth of large-scale and uniform zno nanorod arrays within 3D multichannel monolithic substrates. Cryst. Growth Des. 2013, 13, 3657-3664. [CrossRef]

15. Wang, S.; Ren, Z.; Song, W.; Guo, Y.; Zhang, M.; Suib, S.L.; Gao, P.-X. ZnO/perovskite core-shell nanorod array based monolithic catalysts with enhanced propane oxidation and material utilization efficiency at low temperature. Catal. Today 2015, 258, 549-555. [CrossRef]

16. Ren, Z.; Guo, Y.; Zhang, Z.; Liu, C.; Gao, P.-X. Nonprecious catalytic honeycombs structured with three dimensional hierarchical $\mathrm{Co}_{3} \mathrm{O}_{4}$ nano-arrays for high performance nitric oxide oxidation. J. Mater. Chem. A 2013, 1, 9897-9906. [CrossRef]

17. Ren, Z.; Botu, V.; Wang, S.; Meng, Y.; Song, W.; Guo, Y.; Ramprasad, R.; Suib, S.L.; Gao, P.-X. Monolithically integrated spinel $\mathrm{M}_{\mathrm{x}} \mathrm{Co}_{3-\mathrm{x}} \mathrm{O}_{4}(\mathrm{M}=\mathrm{Co}, \mathrm{Ni}, \mathrm{Zn})$ nanoarray catalysts: Scalable synthesis and cation manipulation for tunable low-temperature $\mathrm{CH} 4$ and $\mathrm{CO}$ oxidation. Angew. Chem. 2014, 53, 7223-7227. [CrossRef] [PubMed]

18. Ren, Z.; Wu, Z.; Song, W.; Xiao, W.; Guo, Y.; Ding, J.; Suib, S.L.; Gao, P.-X. Low temperature propane oxidation over $\mathrm{Co}_{3} \mathrm{O}_{4}$ based nano-array catalysts: Ni dopant effect, reaction mechanism and structural stability. Appl. Catal. B Environ. 2016, 180, 150-160. [CrossRef]

19. Zhang, Z.; Gao, H.; Cai, W.; Liu, C.; Guo, Y.; Gao, P.-X. In situ TPR removal: A generic method for fabricating tubular array devices with mechanical and structural soundness, and functional robustness on various substrates. J. Mater. Chem. 2012, 22, 23098-23105. [CrossRef]

20. Xie, X.; Li, Y.; Liu, Z.-Q.; Haruta, M.; Shen, W. Low-temperature oxidation of $\mathrm{CO}$ catalysed by $\mathrm{Co}_{3} \mathrm{O}_{4}$ nanorods. Nature 2009, 458, 746-749. [CrossRef]

21. $\mathrm{Hu}, \mathrm{L}$.; Peng, Q.; Li, Y. Selective synthesis of $\mathrm{Co}_{3} \mathrm{O}_{4}$ nanocrystal with different shape and crystal plane effect on catalytic property for methane combustion. J. Am. Chem. Soc. 2008, 130, 16136-16137. [CrossRef] [PubMed]

22. Hu, L.; Sun, K.; Peng, Q.; Xu, B.; Li, Y. Surface active sites on $\mathrm{Co}_{3} \mathrm{O}_{4}$ nanobelt and nanocube model catalysts for CO oxidation. Nano Res. 2010, 3, 363-368. [CrossRef]

23. Surendranath, Y.; Kanan, M.W.; Nocera, D.G. Mechanistic studies of the oxygen evolution reaction by a cobalt-phosphate catalyst at neutral ph. J. Am. Chem. Soc. 2010, 132, 16501-16509. [CrossRef] [PubMed]

24. Xie, Y.; Dong, F.; Heinbuch, S.; Rocca, J.J.; Bernstein, E.R. Oxidation reactions on neutral cobalt oxide clusters: Experimental and theoretical studies. Phys. Chem. Chem. Phys. 2010, 12, 947-959. [CrossRef] [PubMed]

25. Chen, S.-Y.; Song, W.; Lin, H.-J.; Wang, S.; Biswas, S.; Mollahosseini, M.; Kuo, C.-H.; Gao, P.-X.; Suib, S.L. Manganese oxide nanoarray-based monolithic catalysts: Tunable morphology and high efficiency for $\mathrm{CO}$ oxidation. ACS Appl. Mater. Interfaces 2016, 8, 7834-7842. [CrossRef] [PubMed]

26. Guo, Y.; Zhang, Z.; Ren, Z.; Gao, H.; Gao, P.-X. Synthesis, characterization and CO oxidation of $\mathrm{TiO}_{2} /(\mathrm{La}, \mathrm{Sr}) \mathrm{MnO}_{3}$ composite nanorod array. Catal. Today 2012, 184, 178-183. [CrossRef]

27. Wu, D.; Zhang, H. Mechanical stability of monolithic catalysts: Scattering of washcoat adhesion and failure mechanism of active material. Ind. Eng. Chem. Res. 2013, 52, 14713-14721. [CrossRef]

28. Wu, D.; Kong, S.; Zhang, H.; Li, Y. Mechanical stability of monolithic catalysts: Factors affecting washcoat adhesion and cohesion during preparation. AIChE J. 2014, 60, 2765-2773. [CrossRef]

29. Hernández-Garrido, J.C.; Gaona, D.; Gómez, D.M.; Gatica, J.M.; Vidal, H.; Sanz, O.; Rebled, J.M.; Peiro, F.; Calvino, J.J. Comparative study of the catalytic performance and final surface structure of $\mathrm{Co}_{3} \mathrm{O}_{4} / \mathrm{La}-\mathrm{CeO}_{2}$ washcoated ceramic and metallic honeycomb monoliths. Catal. Today 2015, 253, 190-198. [CrossRef] 
30. Hernández-Garrido, J.C.; Gómez, D.M.; Gaona, D.; Vidal, H.; Gatica, J.M.; Sanz, O.; Rebled, J.M.; Peiró, F.; Calvino, J.J. Combined (S)TEM-FIB insight into the influence of the preparation method on the final surface structure of $\mathrm{a} \mathrm{Co}_{3} \mathrm{O}_{4} /$ La-modified-CeO $\mathrm{CeO}_{2}$ washcoated monolithic catalyst. J. Phys. Chem. C 2013, 117, 13028-13036. [CrossRef]

31. Cao, A.; Veser, G. Exceptional high-temperature stability through distillation-like self-stabilization in bimetallic nanoparticles. Nat. Mater. 2010, 9, 75-81. [CrossRef]

32. Gandhi, H.; Graham, G.; McCabe, R.W. Automotive exhaust catalysis. J. Catal. 2003, 216, 433-442. [CrossRef]

33. Burton, P.D.; Peterson, E.J.; Boyle, T.J.; Datye, A.K. Synthesis of high surface area ZnO(0001) plates as novel oxide supports for heterogeneous catalysts. Catal. Lett. 2010, 139, 26-32. [CrossRef]

34. Wang, Y.-Z.; Zhao, Y.-X.; Gao, C.-G.; Liu, D.-S. Origin of the high activity and stability of $\mathrm{Co}_{3} \mathrm{O}_{4}$ in low-temperature co oxidation. Catal. Lett. 2008, 125, 134-138. [CrossRef]

35. Zamora, M.; López, T.; Asomoza, M.; Meléndrez, R.; Gómez, R. Alkaline doped $\mathrm{TiO}_{2}$ sol-gel catalysts: Effect of sintering on catalyst activity and selectivity for acetone condensation. Catal. Today 2006, 116, 234-238. [CrossRef]

36. Guo, Y.; Ren, Z.; Xiao, W.; Liu, C.; Sharma, H.; Gao, H.; Mhadeshwar, A.; Gao, P.-X. Robust 3-D configurated metal oxide nano-array based monolithic catalysts with ultrahigh materials usage efficiency and catalytic performance tunability. Nano Energy 2013, 2, 873-881. [CrossRef]

(C) 2017 by the authors. Licensee MDPI, Basel, Switzerland. This article is an open access article distributed under the terms and conditions of the Creative Commons Attribution (CC BY) license (http:/ / creativecommons.org/licenses/by/4.0/). 